\title{
A polarizable continuum model for molecules at spherical diffuse interfaces
}

\author{
Roberto Di Remigio, ${ }^{\text {a) }}$ Krzysztof Mozgawa, Hui Cao, Ville Weijo, and Luca Frediani ${ }^{\text {b) }}$ \\ Department of Chemistry, Centre for Theoretical and Computational Chemistry, UiT, \\ The Arctic University of Norway, N-9037 Troms $\varnothing$, Norway
}

(Received 14 January 2016; accepted 29 February 2016; published online 22 March 2016)

\begin{abstract}
We present an extension of the Polarizable Continuum Model (PCM) to simulate solvent effects at diffuse interfaces with spherical symmetry, such as nanodroplets and micelles. We derive the form of the Green's function for a spatially varying dielectric permittivity with spherical symmetry and exploit the integral equation formalism of the PCM for general dielectric environments to recast the solvation problem into a continuum solvation framework. This allows the investigation of the solvation of ions and molecules in nonuniform dielectric environments, such as liquid droplets, micelles or membranes, while maintaining the computationally appealing characteristics of continuum solvation models. We describe in detail our implementation, both for the calculation of the Green's function and for its subsequent use in the PCM electrostatic problem. The model is then applied on a few test systems, mainly to analyze the effect of interface curvature on solvation energetics. () 2016 AIP Publishing LLC. [http://dx.doi.org/10.1063/1.4943782]
\end{abstract}

\section{INTRODUCTION}

Solvation of ions and molecules at liquid interfaces is of fundamental interest in chemical and biological fields. ${ }^{1-3}$ Understanding how molecules are affected by interfacial environments is therefore crucial in providing insight into many important processes, such as the uptake of pollutants by water droplets ${ }^{4}$ in the atmosphere, or the material transport across the cell membrane. ${ }^{5,6}$ The investigation of the solvation process at surfaces and interfaces has therefore received increasing attention both theoretically and experimentally.

On the experimental side, an important contribution to the molecular structure elucidation at surfaces and interfaces comes from non-linear optical spectroscopy techniques such as Second Harmonic Generation (SHG) ${ }^{7,8}$ and Sum Frequency Generation (SFG). ${ }^{9-11}$ Such techniques are able to target molecules at surfaces and interfaces, because the interfacial environment breaks the inversion symmetry ${ }^{12}$ of the bulk.

On the computational side, most investigations have been carried out by making use of simulation techniques, and in particular Molecular Dynamics (MD) simulations, ${ }^{2,13,14}$ which allow for atomistic details of the whole system providing invaluable insight on the interface structure.

It is however difficult to directly connect molecular dynamic findings with the experimental measurements: MD simulations can be used to obtain the structural information through modeling. On the other hand, a direct comparison of the experimental measurements with theoretical finding will require methods which are able to model the relevant electronic structure parameters: electronic density, transition dipole moments, and polarizabilities of ground and excited states. The natural choice for such studies is in the realm

\footnotetext{
a)Electronic mail: roberto.d.remigio@uit.no

b)Electronic mail: luca.frediani@uit.no
}

of quantum chemistry as the relevant electronic structure parameters can be obtained directly. Modeling the solvent effect for complex environments such as surfaces and interfaces at the quantistic level is however a challenge. The treatment of the whole system is well beyond the capabilities of any modern computing facility, and alternative strategies have to be devised.

When the focus is on the electronic structure of a single molecule or a limited cluster (supermolecule), it is possible to restrict the quantum modeling to the molecule itself and include the environmental effect in a mediated way, either by means of continuum methods ${ }^{15-18}$ or by Quantum Mechanics (QM)/Molecular Mechanics (MM) methods. ${ }^{19-21}$ These approaches have largely and successfully been employed for bulk solutions, but their use for surfaces and interfaces is still limited due to the additional complications brought about by the presence of the interface. For QM/MM methods, it is necessary to generate an adequate number of snapshots from simulations in the presence of the interface which is rather challenging, and it is only very recently that such studies have appeared ${ }^{22-24}$ for planar interfaces. For continuum models, the description of electrostatic effects is rendered more complicated by the variation of the dielectric permittivity which must be accounted for correctly. Moreover, it has been previously shown that for interfaces, it is crucial to consider non-electrostatic interactions in addition to the widely employed electrostatic one. ${ }^{25,26}$

The modeling of the interface itself requires special attention. Although it is relatively easy to model a sharp planar interface, ${ }^{25,27}$ a more correct description should consider diffuse interfaces, where the permittivity changes gradually from the value in one bulk phase to the other: this assumption is physically more reasonable, avoids unphysical divergencies at the interface, and allows for more complicated environments such as membranes or layered environments. This choice is 
also supported by simulations showing that the density profile at the interface is smooth with a sigmoidal shape. ${ }^{28-30}$

The electrostatic problem for planar diffuse interfaces has been originally presented by several authors. ${ }^{31-38}$ However, due to the limited computational resources, the focus was mostly on finding profiles enabling analytical Green's functions. The extension to solvation for QM solutes has later been developed by Frediani et al..$^{39}$ In a recent contribution, Xue and Deng ${ }^{40}$ revisited such a model. They used a quasiharmonic permittivity profile, allowing for a closed, analytical expression of the Green's function.

Nonlocal electrostatic theories offer another possible approach to the modelling of nonuniform dielectric environments. The polarization vector in the medium is defined as the convolution of the applied electric field with a nonlocal susceptibility kernel: $\boldsymbol{P}(\boldsymbol{r})=\int \mathrm{d} \boldsymbol{r}^{\prime} \chi\left(\left|\boldsymbol{r}-\boldsymbol{r}^{\prime}\right|\right) \boldsymbol{E}\left(\boldsymbol{r}^{\prime}\right)$. These approaches, pioneered by Kornyshev and co-workers ${ }^{41-44}$ and later extended to a continuum solvation framework by Basilevsky and co-workers, ${ }^{45-48}$ might offer a more physical description of interfacial phenomena. Knowledge of suitable susceptibility kernels is however still limited, even for bulk solvents, preventing the wide application of nonlocal electrostatic models. ${ }^{18}$

In this contribution, we extend the number of possible environments that can be treated with a continuum solvation approach. Our method can determine the solute-solvent electrostatic interaction for a $\mathrm{QM}$ solute in the presence of an environment with a radially varying permittivity $\varepsilon(r)$. It is in this way possible to rigorously describe the electrostatic part of the solvation effect for media in spherical symmetry, such as nanodroplets, micelles, or even cellular membranes. The formalism is general and it requires only (1) continuity of the permittivity function in any point and (2) an asymptotic constant behavior for large values of $r$. This development makes it possible to study the effect of the droplet size on the solute-solvent electrostatic interaction: although for large systems the planar interface can be a convenient approximation, it is also well known that at the nanoscale, peculiar effects arise and the effect of the size and shape of the droplet are then relevant ones.

The calculation of the Green's function in spherical symmetry for a sharp interface is a textbook example of expansion in spherical harmonics, ${ }^{27,49}$ and has already been adopted in combination with the Polarizable Continuum Model (PCM) by Delgado et al. ${ }^{50}$ Similar models have been employed by Deng, ${ }^{51-53}$ who has extended them to ellipsoidal geometries and layered models. Xue and Deng ${ }^{54,55}$ have later on generalized the model to diffuse interfaces for quasiharmonic dielectric profiles, such that the solution can then be expressed in terms of a series expansion, rather than direct numerical integration, which is the approach we have followed instead. By making use of a quasiharmonic permittivity profile, Deng presented also a strategy for the simulation of spherical quantum dots in the presence of general, spatially varying permittivities. ${ }^{53}$

It is important to emphasize that to achieve a correct picture of solvation at surfaces and interfaces, it has been demonstrated that electrostatics is not enough and nonelectrostatic contributions need to be accounted for. ${ }^{26}$ In this contribution, we limit however ourselves to considering the electrostatic effect to focus on the main novelty of our work, which is the inclusion of electrostatic solvation at spherical diffuse interfaces for quantum chemical modeling and arbitrary dielectric profile.

In Section II, the theory for the determination of the Green's function is presented. After a brief description of the implementation (Section III), we illustrate the application of our methodology (Section IV) on inorganic ions and organic chromophores. We summarize our conclusions in Section V.

\section{THEORY}

\section{A. Integral Equation Formalism (IEF)-PCM}

Dielectric Continuum (DC) models provide a simple, yet effective strategy to treat solvent effects on molecular systems by treating implicitly the degrees of freedom relative to the solvent molecules forming the environment. The solvent is replaced by a structureless continuum described by its bulk properties, such as the dielectric permittivity $\varepsilon$. The solute is then placed in a cavity $C \subset \mathbb{R}^{3}$ with boundary $\partial C$ inside this continuum. The calculation of the mutual polarization between the solute charge density $\rho$ and the infinite continuum is performed by solving the classical Poisson problem for the electrostatic potential $u(\boldsymbol{r})$,

$$
\left\{\begin{array}{l}
\nabla^{2} u(\boldsymbol{r})=-\rho(\boldsymbol{r}) \quad \forall \boldsymbol{r} \in C \\
\nabla \cdot[\varepsilon \nabla u(\boldsymbol{r})]=0 \quad \forall \boldsymbol{r} \notin C \\
\lim _{|\boldsymbol{r}| \rightarrow \partial C^{+}} u(\boldsymbol{r})=\lim _{|\boldsymbol{r}| \rightarrow \partial C^{-}} u(\boldsymbol{r}) \\
\varepsilon \lim _{|\boldsymbol{r}| \rightarrow \partial C^{+}} \frac{\partial u(\boldsymbol{r})}{\partial \boldsymbol{n}}=\lim _{|\boldsymbol{r}| \rightarrow \partial C^{-}} \frac{\partial u(\boldsymbol{r})}{\partial \boldsymbol{n}}
\end{array}\right.
$$

where the last two equations represent the boundary conditions: the requirement of continuity for the electrostatic potential across boundary and the jump condition on its normal derivative. ${ }^{27}$ The values at the boundary are defined in terms of trace operators,${ }^{56}$ i. e., in terms of limits taken from the outside (+ subscript) or the inside (- subscript) of the cavity boundary.

The Poisson problem defined by Eq. (1) can be solved by formulating it as a boundary integral equation. ${ }^{57}$ First, the electrostatic potential is separated into the Newton $\mathcal{N}_{\rho}=\int_{C} \mathrm{~d} \boldsymbol{r}^{\prime} \frac{\rho\left(r^{\prime}\right)}{\left|\boldsymbol{r}-\boldsymbol{r}^{\prime}\right|}$ and the reaction potentials $\xi$,

$$
u(\boldsymbol{r})=\mathcal{N}_{\rho}+\xi .
$$

The reaction potential is then represented in terms of an apparent surface charge (ASC) $\sigma(\boldsymbol{s})$,

$$
\xi(\boldsymbol{r})=\int_{\partial C} \mathrm{~d} \boldsymbol{s} \frac{\sigma(\boldsymbol{s})}{|\boldsymbol{r}-\boldsymbol{s}|},
$$

such that the polarization in the continuum is entirely described by a surface charge, a scalar function of the surface coordinate $\boldsymbol{s}$. The ASC is obtained as the unique solution of the following integral equation: ${ }^{17}$

$$
\left[\left(\frac{1}{2}-\mathcal{D}_{\mathrm{e}}\right) \mathcal{S}_{\mathrm{i}}+\mathcal{S}_{\mathrm{e}}\left(\frac{1}{2}+\mathcal{D}_{\mathrm{i}}^{\dagger}\right)\right] \sigma=\left(\mathcal{D}_{\mathrm{e}}-\frac{1}{2}\right) \mathcal{N}_{\rho}-\mathcal{S}_{\mathrm{e}} \frac{\partial \mathcal{N}_{\rho}}{\partial \boldsymbol{n}},
$$


also known as the IEF equation.

The integral operators in the IEF-PCM equation are expressed in terms of the components of the Calderón projector,

$$
\begin{aligned}
& \mathcal{S}_{\star} f(\boldsymbol{s})=\int_{\partial C} \mathrm{~d} \boldsymbol{s}^{\prime} G_{\star}\left(\boldsymbol{s}, \boldsymbol{s}^{\prime}\right) f\left(\boldsymbol{s}^{\prime}\right), \\
& \mathcal{D}_{\star} f(\boldsymbol{s})=\int_{\partial C} \mathrm{~d} \boldsymbol{s}^{\prime} \varepsilon\left[\nabla_{\boldsymbol{s}^{\prime}} G_{\star}\left(\boldsymbol{s}, \boldsymbol{s}^{\prime}\right) f\left(\boldsymbol{s}^{\prime}\right)\right] \cdot \boldsymbol{n}_{\boldsymbol{s}^{\prime}}, \\
& \mathcal{D}_{\star}^{\dagger} f(\boldsymbol{s})=\int_{\partial C} \mathrm{~d} \boldsymbol{s}^{\prime} \varepsilon\left[\nabla_{s} G_{\star}\left(\boldsymbol{s}, \boldsymbol{s}^{\prime}\right) f\left(\boldsymbol{s}^{\prime}\right)\right] \cdot \boldsymbol{n}_{\boldsymbol{s}} .
\end{aligned}
$$

The internal and external Calderón projectors are here marked by the placeholder subscript $\star \in\{\mathrm{i}, \mathrm{e}\}$.

As already noted elsewhere ${ }^{39,58}$ knowledge of the Green's function for the differential operators involved in the Poisson problem guarantees that the IEF-PCM approach is applicable also for dielectric environments that are not uniform and homogeneous. This is in fact the aim of the Sec. II B, where the Green's function for a position-dependent permittivity in spherical symmetry will be derived. The solution of Eq. (5) is achieved using the boundary element method (BEM), a procedure detailed in Section II D. Note that Hartree atomic units have been used and will be used throughout the text.

\section{B. Green's function in spherical symmetry}

The general electrostatic Green's function $G\left(\boldsymbol{r}, \boldsymbol{r}^{\prime}\right)$ for a system with a position-dependent dielectric constant, $\varepsilon(\boldsymbol{r})$, is formally defined as the solution to the inhomogeneous differential equation,

$$
\nabla \cdot\left[\varepsilon(\boldsymbol{r}) \nabla G\left(\boldsymbol{r}, \boldsymbol{r}^{\prime}\right)\right]=-4 \pi \delta\left(\boldsymbol{r}-\boldsymbol{r}^{\prime}\right)
$$

which can be rearranged as ${ }^{27,59}$

$$
\varepsilon(\boldsymbol{r}) \nabla^{2} G\left(\boldsymbol{r}, \boldsymbol{r}^{\prime}\right)+\nabla \varepsilon(\boldsymbol{r}) \cdot \nabla G\left(\boldsymbol{r}, \boldsymbol{r}^{\prime}\right)=-4 \pi \delta\left(\boldsymbol{r}-\boldsymbol{r}^{\prime}\right) .
$$

We assume that the permittivity has spherical symmetry. It is thus convenient to reformulate Eq. (7) in a spherical coordinate system- $r, \theta, \phi$-with the origin in the center of the spherical droplet. Given $r \in[0,+\infty)$ as the radial distance, $\theta \in[0, \pi]$ as the polar angle and $\phi \in[0,2 \pi)$ as the azimuth, Eq. (7) can be rewritten as

$$
\begin{aligned}
\frac{1}{r^{2}} & {\left[\frac{\partial}{\partial r}\left(r^{2} \frac{\partial G}{\partial r}\right)+\frac{1}{\sin \theta} \frac{\partial}{\partial \theta}\left(\sin \theta \frac{\partial G}{\partial \theta}\right)+\frac{1}{\sin ^{2} \theta} \frac{\partial^{2} G}{\partial \phi^{2}}\right] } \\
& +\lambda_{\varepsilon}(r) \frac{\partial G}{\partial r}=-\frac{4 \pi \delta\left(\boldsymbol{r}-\boldsymbol{r}^{\prime}\right)}{\varepsilon(r)},
\end{aligned}
$$

where the weighted dielectric distribution,

$$
\lambda_{\varepsilon}(r)=\frac{\mathrm{d} \varepsilon(r)}{\mathrm{d} r} \frac{1}{\varepsilon(r)},
$$

was introduced. Further regularity assumptions on the permittivity profile will be detailed in the Appendix, but it can already be noted that it has to be nonzero on the whole domain to be acceptable.

We now consider $G\left(\boldsymbol{r}, \boldsymbol{r}^{\prime}\right)$ as a function of $\boldsymbol{r}$ and expand it in spherical harmonics, ${ }^{59}$

$$
G\left(\boldsymbol{r}, \boldsymbol{r}^{\prime}\right)=\sum_{\ell m} \bar{y}_{\ell}^{m}\left(\theta^{\prime}, \phi^{\prime}\right) R_{\ell}\left(r, r^{\prime}\right), \mathcal{Y}_{\ell}^{m}(\theta, \phi)
$$

where $\mathcal{Y}_{\ell}^{m}(\theta, \phi)$ is the spherical harmonic function of degree $\ell$ and order $m$ and $\bar{y}_{\ell}^{m}\left(\theta^{\prime}, \phi^{\prime}\right)$ is its complex conjugate. The shorthand notation $\sum_{\ell m}=\sum_{\ell=0}^{\infty} \sum_{m=-\ell}^{+\ell}$ was introduced. The spherical harmonics are eigenfunctions of the angular operator, ${ }^{60}$

$$
\begin{gathered}
-\left[\frac{1}{\sin \theta} \frac{\partial}{\partial \theta}\left(\sin \theta \frac{\partial}{\partial \theta}\right)+\frac{1}{\sin ^{2} \theta} \frac{\partial^{2}}{\partial \phi^{2}}\right] y_{\ell}^{m}(\theta, \phi) \\
=\ell(\ell+1) y_{\ell}^{m}(\theta, \phi) .
\end{gathered}
$$

Substitution of this expansion in Eq. (8) together with the expansion of the $\delta$-function on the right-hand side (RHS),

$$
\delta\left(\boldsymbol{r}-\boldsymbol{r}^{\prime}\right)=\frac{1}{r^{2}} \delta\left(r-r^{\prime}\right) \sum_{\ell m} \overline{\boldsymbol{y}}_{\ell}^{m}\left(\theta^{\prime}, \phi^{\prime}\right) \boldsymbol{Y}_{\ell}^{m}(\theta, \phi),
$$

yields

$$
\begin{aligned}
\sum_{\ell m}( & \left(\frac{1}{r} \frac{\mathrm{d}^{2}\left(r R_{\ell}\right)}{\mathrm{d} r^{2}}-\frac{\ell(\ell+1)}{r^{2}} R_{\ell}+\lambda_{\varepsilon}(r) \frac{\mathrm{d} R_{\ell}}{\mathrm{d} r}\right) \\
& \times \overline{\mathcal{Y}}_{l}^{m}\left(\theta^{\prime}, \phi^{\prime}\right) \mathcal{Y}_{\ell}^{m}(\theta, \phi) \\
& =-\frac{4 \pi}{r^{2}} \frac{1}{\varepsilon(r)} \delta\left(r-r^{\prime}\right) \sum_{\ell m} \bar{y}_{l}^{m}\left(\theta^{\prime}, \phi^{\prime}\right) \mathcal{Y}_{\ell}^{m}(\theta, \phi) .
\end{aligned}
$$

By virtue of the completeness of the spherical harmonics functions, we are left with the radial equation,

$$
\begin{aligned}
\frac{\mathrm{d}^{2} R_{\ell}}{\mathrm{d} r^{2}} & +\left(\frac{2}{r}+\lambda_{\varepsilon}(r)\right) \frac{\mathrm{d} R_{\ell}}{\mathrm{d} r}-\frac{\ell(\ell+1)}{r^{2}} R_{\ell} \\
& =-\frac{4 \pi}{r^{2}} \frac{1}{\varepsilon(r)} \delta\left(r-r^{\prime}\right) .
\end{aligned}
$$

The radial Eq. (14) is a second-order inhomogeneous ordinary differential equation (ODE). It is useful to note that, in the absence of the dielectric inhomogeneity due to the diffuse layer, the radial equation would be

$$
\frac{\mathrm{d}^{2} R_{\ell}}{\mathrm{d} r^{2}}+\frac{2}{r} \frac{\mathrm{d} R_{\ell}}{\mathrm{d} r}-\frac{\ell(\ell+1)}{r^{2}} R_{\ell}=-\frac{4 \pi}{r^{2}} \delta\left(r-r^{\prime}\right) .
$$

Eq. (14) has an additional first-order term $-\lambda_{\varepsilon} \frac{\mathrm{d} R_{\ell}}{\mathrm{d} r}$-on the left-hand side (LHS). Furthermore, the source inhomogeneity on the RHS is scaled by the value of the dielectric profile at the source point $\varepsilon(r)$.

To solve Eq. (14) for the radial part of the Green's function, we first solve the associated homogeneous equation,

$$
\frac{\mathrm{d}^{2} R_{\ell}}{\mathrm{d} r^{2}}+\left(\frac{2}{r}+\lambda_{\varepsilon}(r)\right) \frac{\mathrm{d} R_{\ell}}{\mathrm{d} r}-\frac{\ell(\ell+1)}{r^{2}} R_{\ell}=0
$$

and then integrate the RHS source inhomogeneity to find a particular solution. ${ }^{60}$ Eq. (16) admits two linearly independent solutions, ${ }^{60} u_{\ell}$ and $v_{\ell}$, whose asymptotic behavior is fixed to the same as for the solution to the radial equation in vacuo, ${ }^{27,59}$

$$
u_{\ell}=r^{\ell}, \quad v_{\ell}=\frac{1}{r^{\ell+1}} .
$$

This is a valid assumption for profiles represented by a sigmoidal functional form, see the Appendix. The constraint on the functional form of the two linearly independent solutions defines also the initial values for the solution of 
Eq. (16),

$$
\left\{\begin{array}{l}
\lim _{r \rightarrow 0} u_{\ell}=0 \\
\lim _{r \rightarrow \infty} u_{\ell}=\infty
\end{array}, \quad\left\{\begin{array}{l}
\lim _{r \rightarrow 0} v_{\ell}=\infty \\
\lim _{r \rightarrow \infty} v_{\ell}=0
\end{array} .\right.\right.
$$

The most general radial solution will be a linear combination of $u_{\ell}$ and $v_{\ell}$. The coefficients are determined by the RHS source inhomogeneity and will be different for the cases $r<r^{\prime}$ and $r>r^{\prime}$,

$$
R_{\ell}=\left\{\begin{array}{l}
\left.\left.c_{\ell}^{(1)}\right|_{r^{\prime}} u_{\ell}\right|_{r}+\left.\left.c_{\ell}^{(2)}\right|_{r^{\prime}} v_{\ell}\right|_{r}, \text { for } r<r^{\prime} \\
\left.\left.c_{\ell}^{(3)}\right|_{r^{\prime}} u_{\ell}\right|_{r}+\left.\left.c_{\ell}^{(4)}\right|_{r^{\prime}} v_{\ell}\right|_{r}, \text { for } r>r^{\prime}
\end{array} .\right.
$$

Notice that the coefficients are unknown functions of the second radial variable and the function trace notation to denote evaluation of a function at a given radial point,

$$
\left.f\right|_{r}=f(r),
$$

was introduced. Due to the requirement that the Green's function be finite at the origin and at infinity, we can already determine that $\left.c_{\ell}^{(2)}\right|_{r^{\prime}}=0$ and $\left.c_{\ell}^{(3)}\right|_{r^{\prime}}=0$. The Green's function has thus the following form:

$$
G\left(\boldsymbol{r}, \boldsymbol{r}^{\prime}\right)=\left\{\begin{array}{l}
\left.\left.\sum_{\ell=0}^{\infty} \frac{2 \ell+1}{4 \pi} c_{\ell}^{(1)}\right|_{r^{\prime}} u_{\ell}\right|_{r} P_{\ell}(\cos \gamma), \text { for } r<r^{\prime} \\
\left.\left.\sum_{\ell=0}^{\infty} \frac{2 \ell+1}{4 \pi} c_{\ell}^{(4)}\right|_{r^{\prime}} v_{\ell}\right|_{r} P_{\ell}(\cos \gamma), \text { for } r>r^{\prime}
\end{array}\right.
$$

The spherical harmonics addition theorem ${ }^{60}$ was used and the argument to the Legendre polynomial is

$\cos \gamma=\frac{\boldsymbol{r} \cdot \boldsymbol{r}^{\prime}}{|\boldsymbol{r}|\left|\boldsymbol{r}^{\prime}\right|}=\cos \theta \cos \theta^{\prime}+\sin \theta \sin \theta^{\prime} \cos \left(\phi-\phi^{\prime}\right)$.

The coefficients can be found by imposing the continuity of the Green's function for $r=r^{\prime}$ and $\gamma \neq 0$,

$$
\left.\left.c_{\ell}^{(1)}\right|_{r^{\prime}} u_{\ell}\right|_{r^{\prime}}=\left.c_{\ell}^{(4)}\right|_{r^{\prime}},\left.v_{\ell}\right|_{r^{\prime}},
$$

and by integrating Eq. (14) in a vanishingly small volume element centered around $r^{\prime}$,

$$
\begin{aligned}
\lim _{\delta \rightarrow 0} & \int_{r^{\prime}-\delta}^{r^{\prime}+\delta} \mathrm{d} r\left\{\frac{\mathrm{d}^{2} R_{\ell}}{\mathrm{d} r^{2}}+\left(\frac{2}{r}+\lambda_{\varepsilon}(r)\right) \frac{\mathrm{d} R_{\ell}}{\mathrm{d} r}-\frac{\ell(\ell+1)}{r^{2}} R_{\ell}\right\} \\
& =\lim _{\delta \rightarrow 0} \int_{r^{\prime}-\delta}^{r^{\prime}+\delta} \mathrm{d} r\left\{-\frac{4 \pi}{r^{2}} \frac{1}{\varepsilon(r)} \delta\left(r-r^{\prime}\right)\right\} .
\end{aligned}
$$

The RHS is easily integrated to yield

$$
\lim _{\delta \rightarrow 0} \int_{r^{\prime}-\delta}^{r^{\prime}+\delta} \mathrm{d} r\left\{-\frac{4 \pi}{r^{2}} \frac{1}{\varepsilon(r)} \delta\left(r-r^{\prime}\right)\right\}=-\frac{4 \pi}{r^{\prime 2}} \frac{1}{\left.\varepsilon\right|_{r^{\prime}}} .
$$

For the LHS, we have

$$
\begin{aligned}
\lim _{\delta \rightarrow 0} & \int_{r^{\prime}-\delta}^{r^{\prime}+\delta} \mathrm{d} r\left\{\frac{\mathrm{d}^{2} R_{\ell}}{\mathrm{d} r^{2}}+\left(\frac{2}{r}+\lambda_{\varepsilon}(r)\right) \frac{\mathrm{d} R_{\ell}}{\mathrm{d} r}-\frac{\ell(\ell+1)}{r^{2}} R_{\ell}\right\} \\
=\left.\frac{\mathrm{d} R_{\ell}}{\mathrm{d} r}\right|_{r^{\prime}-\delta} ^{r^{\prime}+\delta}, &
\end{aligned}
$$

and the remaining terms in (24) are in fact zero by virtue of the mean value theorem for integration,

$$
\begin{aligned}
\lim _{\delta \rightarrow 0} \int_{r^{\prime}-\delta}^{r^{\prime}+\delta} \mathrm{d} r\left\{\left(\frac{2}{r}+\lambda_{\varepsilon}(r)\right) \frac{\mathrm{d} R_{\ell}}{\mathrm{d} r}-\frac{\ell(\ell+1)}{r^{2}} R_{\ell}\right\} \\
=\lim _{\delta \rightarrow 0} 2 \delta\left\langle\left(\frac{2}{r}+\lambda_{\varepsilon}(r)\right) \frac{\mathrm{d} R_{\ell}}{\mathrm{d} r}-\frac{\ell(\ell+1)}{r^{2}} R_{\ell}\right\rangle=0,
\end{aligned}
$$

where angle brackets were used to indicate the average value of the integrand function. We thus obtain a system of linear equations from Eqs. (23) and (24),

$$
\left\{\begin{array}{l}
\left.\left.c_{\ell}^{(1)}\right|_{r^{\prime}} u_{\ell}\right|_{r^{\prime}}-\left.\left.c_{\ell}^{(4)}\right|_{r^{\prime}} v_{\ell}\right|_{r^{\prime}}=0 \\
\left.\left.c_{\ell}^{(1)}\right|_{r^{\prime}} \frac{\mathrm{d} u_{\ell}}{\mathrm{d} r}\right|_{r^{\prime}}-\left.\left.c_{\ell}^{(4)}\right|_{r^{\prime}} \frac{\mathrm{d} v_{\ell}}{\mathrm{d} r}\right|_{r^{\prime}}=\frac{4 \pi}{r^{\prime 2}} \frac{1}{\varepsilon\left(r^{\prime}\right)}
\end{array}\right.
$$

whose solution fully determines the radial part of the Green's function,

$$
\left.c_{\ell}^{(1)}\right|_{r^{\prime}}=-\frac{4 \pi}{r^{\prime 2}} \frac{1}{\left.\varepsilon\right|_{r^{\prime}}} \frac{\left.v_{\ell}\right|_{r^{\prime}}}{\left.W_{\ell}\right|_{r^{\prime}}},\left.\quad c_{\ell}^{(4)}\right|_{r^{\prime}}=-\frac{4 \pi}{r^{\prime 2}} \frac{1}{\left.\varepsilon\right|_{r^{\prime}}} \frac{\left.u_{\ell}\right|_{r^{\prime}}}{\left.W_{\ell}\right|_{r^{\prime}}} .
$$

The coefficients are defined in terms of the Wronskian,

$$
\left.W_{\ell}\right|_{r^{\prime}}=\left.\left.\frac{\mathrm{d} v_{\ell}}{\mathrm{d} r}\right|_{r^{\prime}} u_{\ell}\right|_{r^{\prime}}-\left.\left.\frac{\mathrm{d} u_{\ell}}{\mathrm{d} r}\right|_{r^{\prime}} v_{\ell}\right|_{r^{\prime}}
$$

Eventually, the Green's function reads

$$
\begin{aligned}
G\left(\boldsymbol{r}, \boldsymbol{r}^{\prime}\right) & =\sum_{\ell=0}^{\infty} G_{\ell}\left(\boldsymbol{r}, \boldsymbol{r}^{\prime}\right) \\
& =\left\{\begin{array}{l}
\sum_{\ell=0}^{\infty} \frac{-(2 \ell+1)}{\left.r^{\prime 2} \varepsilon\right|_{r^{\prime}}} \frac{\left.\left.u_{\ell}\right|_{r} v_{\ell}\right|_{r^{\prime}}}{\left.W_{\ell}\right|_{r^{\prime}}} P_{\ell}(\cos \gamma), \text { for } r<r^{\prime} \\
\sum_{\ell=0}^{\infty} \frac{-(2 \ell+1)}{\left.r^{\prime 2} \varepsilon\right|_{r^{\prime}}} \frac{\left.\left.u_{\ell}\right|_{r^{\prime}} v_{\ell}\right|_{r}}{\left.W_{\ell}\right|_{r^{\prime}}} P_{\ell}(\cos \gamma), \text { for } r>r^{\prime}
\end{array}\right.
\end{aligned}
$$

\section{Separation of the Coulomb singularity}

The working expression for the Green's function in Eq. (31) includes the Coulomb singularity arising when source and probe point coalesce. It is important to isolate such a singularity, for two reasons. First of all, the presence of the singularity in the components $G_{\ell}\left(\boldsymbol{r}, \boldsymbol{r}^{\prime}\right)$ would make the summation slowly converging. Moreover, since the boundary element representation of the integral operators will be obtained by a collocation method, ${ }^{17}$ isolating the singularity avoids instabilities in the evaluation of the diagonal matrix elements, i.e., the self-interaction at the collocation points. ${ }^{61,62}$

Formally, we can rewrite the Green's function as the sum of singular and non-singular terms,

$$
G\left(\boldsymbol{r}, \boldsymbol{r}^{\prime}\right)=\frac{1}{C\left(\boldsymbol{r}, \boldsymbol{r}^{\prime}\right)\left|\boldsymbol{r}-\boldsymbol{r}^{\prime}\right|}+G_{\mathrm{img}}\left(\boldsymbol{r}, \boldsymbol{r}^{\prime}\right) .
$$

The $C\left(\boldsymbol{r}, \boldsymbol{r}^{\prime}\right)$ factor accounts for the variation of the permittivity and "modulates" the Coulomb singularity according to the dielectric environment. To preserve the physical meaning of the Green's function, we require that

$$
\lim _{\left|\boldsymbol{r}-\boldsymbol{r}^{\prime}\right| \rightarrow 0} C\left(\boldsymbol{r}, \boldsymbol{r}^{\prime}\right)=\varepsilon(r)
$$


such that the Green's function is that for a uniform dielectric with constant permittivity,

$$
\lim _{\left|\boldsymbol{r}-\boldsymbol{r}^{\prime}\right| \rightarrow 0} G\left(\boldsymbol{r}, \boldsymbol{r}^{\prime}\right)=\frac{1}{\varepsilon(r)\left|\boldsymbol{r}-\boldsymbol{r}^{\prime}\right|} .
$$

To derive a useful expression for the separation coefficient, we use the exact expansion of the Coulomb singularity, ${ }^{27}$

$$
\begin{aligned}
\frac{1}{\left|\boldsymbol{r}-\boldsymbol{r}^{\prime}\right|} & =\sum_{\ell=0}^{\infty} g_{\ell}\left(\boldsymbol{r}, \boldsymbol{r}^{\prime}\right) \\
& =\left\{\begin{array}{l}
\frac{1}{r^{\prime}} \sum_{\ell=0}^{\infty}\left(\frac{r}{r^{\prime}}\right)^{\ell} P_{\ell}(\cos \gamma), \text { for } r<r^{\prime}, \\
\frac{1}{r} \sum_{\ell=0}^{\infty}\left(\frac{r^{\prime}}{r}\right)^{\ell} P_{\ell}(\cos \gamma), \text { for } r>r^{\prime}
\end{array}\right.
\end{aligned}
$$

and obtain for the image Green's function,

$$
\tilde{G}_{\mathrm{img}}\left(\boldsymbol{r}, \boldsymbol{r}^{\prime}\right)=\sum_{\ell=0}^{\infty}\left\{G_{\ell}\left(\boldsymbol{r}, \boldsymbol{r}^{\prime}\right)-\frac{1}{C\left(\boldsymbol{r}, \boldsymbol{r}^{\prime}\right)} g_{\ell}\left(\boldsymbol{r}, \boldsymbol{r}^{\prime}\right)\right\} .
$$

We are here using $\tilde{G}_{\text {img }}\left(\boldsymbol{r}, \boldsymbol{r}^{\prime}\right)$ because the image contribution so defined contains spurious contribution that have to be renormalized. At large $\ell$, the Coulomb singularity dominates over the image part,

$$
\lim _{\ell \rightarrow \infty} \tilde{G}_{\mathrm{img}}\left(\boldsymbol{r}, \boldsymbol{r}^{\prime}\right)=0=\lim _{\ell \rightarrow \infty} G_{\mathrm{img}}\left(\boldsymbol{r}, \boldsymbol{r}^{\prime}\right),
$$

thus

$$
\lim _{\ell \rightarrow \infty}\left\{G_{\ell}\left(\boldsymbol{r}, \boldsymbol{r}^{\prime}\right)-\frac{1}{C\left(\boldsymbol{r}, \boldsymbol{r}^{\prime}\right)} g_{\ell}\left(\boldsymbol{r}, \boldsymbol{r}^{\prime}\right)\right\}=0
$$

leading to

$$
C\left(\boldsymbol{r}, \boldsymbol{r}^{\prime}\right)=\left\{\begin{array}{l}
\frac{1}{r^{\prime}} \lim _{\ell \rightarrow \infty}\left(\frac{r}{r^{\prime}}\right)^{\ell} \frac{P_{\ell}(\cos \gamma)}{G_{\ell}\left(\boldsymbol{r}, \boldsymbol{r}^{\prime}\right)}, \text { for } r<r^{\prime}, \\
\frac{1}{r} \lim _{\ell \rightarrow \infty}\left(\frac{r^{\prime}}{r}\right)^{\ell} \frac{P_{\ell}(\cos \gamma)}{G_{\ell}\left(\boldsymbol{r}, \boldsymbol{r}^{\prime}\right)}, \text { for } r>r^{\prime} .
\end{array}\right.
$$

Once the coefficient is calculated, the leading monopole term $(\ell=0$ term) in the Legendre polynomial expansion of the Green's function can be expressed in its exact singular form. This term must thus be excluded a posteriori from the summation of the image contribution, by starting the angular momentum summation from $\ell=1$,

$$
G_{\text {img }}\left(\boldsymbol{r}, \boldsymbol{r}^{\prime}\right)=\sum_{\ell=1}^{\infty}\left(G_{\ell}\left(\boldsymbol{r}, \boldsymbol{r}^{\prime}\right)-\frac{1}{C\left(\boldsymbol{r}, \boldsymbol{r}^{\prime}\right)\left|\boldsymbol{r}-\boldsymbol{r}^{\prime}\right|}\right) .
$$

This avoids the appearance of an unphysical monopole contribution in the image potential, which is consistent with the analytic expression of the Green's function for a spherically symmetric dielectric permittivity with sharp boundary. ${ }^{49}$

\section{Collocation BEM for the IEF-PCM equation}

The numerical solution of boundary integral equations, such as the IEF-PCM Eq. (4), can be achieved by the application of the BEM. A discretization of the molecular cavity boundary is necessary. ${ }^{62}$ The discretization of the molecular surface into finite elements leads to a corresponding discretization of both the integral operators and the functions in Eq. (4). Different strategies are available to perform the discretization: the collocation or the Galerkin approach. ${ }^{63}$ The final result is the transformation of the original integral equation to a system of linear equations whose dimension relates to the number of finite elements generated by discretizing the boundary. For general integral equations and discretization schemes, a dense system matrix is obtained. Moreover, the discretization scheme might not, in general, preserve the symmetry property of the original integral equation.

In this paper, we apply the traditional centroid collocation method $^{18,62}$ on the cavity boundary discretization obtained via the GePol algorithm. ${ }^{62,64-68}$ In this algorithm, the cavity is made of interlocking spheres, not all of them necessarily centered on the atoms. The surface is then discretized by means of $N_{\text {ts }}$ small triangles $\Delta_{i}$ whose centroid defines the collocation point. Once the centroid is properly defined, functions and operators are discretized by applying a one-point quadrature rule in the finite element centroid. Eq. (4) is thus recast as

$$
q=T^{-1} R v,
$$

where $\boldsymbol{q}$ and $\boldsymbol{v}$ are vectors of dimension $N_{\text {ts }}$ collecting the values of the ASC and molecular electrostatic potential (MEP) at the collocation points. The matrices representing the boundary integral operators are

$$
\begin{aligned}
& \boldsymbol{T}=\left(2 \pi \boldsymbol{I}-\boldsymbol{D}_{\mathrm{e}} \boldsymbol{A}\right) \boldsymbol{S}_{\mathrm{i}}+\boldsymbol{S}_{\mathrm{e}}\left(2 \pi \boldsymbol{I}+\boldsymbol{A} \boldsymbol{D}_{\mathrm{i}}^{\dagger}\right), \\
& \boldsymbol{R}=\left(2 \pi \boldsymbol{I}-\boldsymbol{D}_{\mathrm{e}} \boldsymbol{A}\right)-\boldsymbol{S}_{\mathrm{e}} \boldsymbol{S}_{\mathrm{i}}^{-1}\left(2 \pi \boldsymbol{I}-\boldsymbol{D}_{\mathrm{i}} \boldsymbol{A}\right),
\end{aligned}
$$

where $\boldsymbol{I}$ is the $N_{\mathrm{ts}}$-dimensional identity matrix while the other matrices are defined as

$$
\begin{gathered}
\boldsymbol{A}=\operatorname{diag}\left(\cdots a_{i}=\operatorname{area}\left(\Delta_{i}\right) \cdots\right), \\
S_{i i, \mathrm{i}}=k \sqrt{\frac{4 \pi}{a_{i}}}, \quad S_{i j, \mathrm{i}}=\frac{1}{\left|\boldsymbol{s}_{i}-\boldsymbol{s}_{j}\right|} \quad(i \neq j), \\
D_{i i, \mathrm{i}}=-k \sqrt{\frac{\pi}{a_{i}}} \frac{1}{R_{i}}, \quad D_{i j, \mathrm{i}}=\frac{\left(\boldsymbol{s}_{i}-\boldsymbol{s}_{j}\right) \cdot \boldsymbol{n}_{j}}{\left|\boldsymbol{s}_{i}-\boldsymbol{s}_{j}\right|^{3}} \quad(i \neq j), \\
S_{i i, \mathrm{e}}=\frac{S_{i i, \mathrm{i}}}{C\left(\boldsymbol{s}_{i}, \boldsymbol{s}_{i}\right)}+G_{\mathrm{img}}\left(\boldsymbol{s}_{i}, \boldsymbol{s}_{i}\right), \\
S_{i j, \mathrm{e}}=\frac{1}{C\left(\boldsymbol{s}_{i}, \boldsymbol{s}_{j}\right)\left|\boldsymbol{s}_{i}-\boldsymbol{s}_{j}\right|}+G_{\mathrm{img}}\left(\boldsymbol{s}_{i}, \boldsymbol{s}_{j}\right) \quad(i \neq j),
\end{gathered}
$$

$$
\begin{aligned}
& D_{i i, \mathrm{e}}=\varepsilon\left(\boldsymbol{s}_{i}\right)\left[\frac{D_{i i, \mathrm{i}}}{C\left(\boldsymbol{s}_{i}, \boldsymbol{s}_{i}\right)}-S_{i i, \mathrm{i}} \frac{\nabla C\left(\boldsymbol{s}_{i}, \boldsymbol{s}_{i}\right) \cdot \boldsymbol{n}_{i}}{C^{2}\left(\boldsymbol{s}_{i}, \boldsymbol{s}_{i}\right)}+\nabla G_{\mathrm{img}}\left(\boldsymbol{s}_{i}, \boldsymbol{s}_{i}\right) \cdot \boldsymbol{n}_{i}\right] \\
& D_{i j, \mathrm{e}}=\varepsilon\left(\boldsymbol{s}_{j}\right)\left[\frac{1}{C\left(\boldsymbol{s}_{i}, \boldsymbol{s}_{j}\right)} \frac{\left(\boldsymbol{s}_{i}-\boldsymbol{s}_{j}\right) \cdot \boldsymbol{n}_{j}}{\left|\boldsymbol{s}_{i}-\boldsymbol{s}_{j}\right|^{3}}-\frac{\nabla C\left(\boldsymbol{s}_{i}, \boldsymbol{s}_{j}\right) \cdot \boldsymbol{n}_{j}}{C^{2}\left(\boldsymbol{s}_{i}, \boldsymbol{s}_{j}\right)} \frac{1}{\left|\boldsymbol{s}_{i}-\boldsymbol{s}_{j}\right|}+\nabla G_{\mathrm{img}}\left(\boldsymbol{s}_{i}, \boldsymbol{s}_{j}\right) \cdot \boldsymbol{n}_{j}\right] \quad(i \neq j)
\end{aligned}
$$


in terms of the centroids $\boldsymbol{s}_{i}, \boldsymbol{s}_{j}$, the finite elements areas, and their curvatures $R_{i}$. The factor $k=1.07$ is introduced to achieve a better precision on the diagonal elements. ${ }^{18}$

\section{E. Coupling to quantum mechanics}

To account for the mutual polarization between the classical continuum and the quantum mechanical molecular charge distribution, we need to include a suitable operator in the usual vacuum Hamiltonian,

$$
H=H_{0}+V_{\sigma \rho}[\rho] .
$$

The PCM operator $V_{\sigma \rho}[\rho]$ depends on the functional form of the solute charge density and thus introduces a nonlinear coupling in the quantum mechanical problem. The ground state of the PCM Hamiltonian can be found by minimization of a proper functional ${ }^{69}$ whose physical interpretation is that of a free energy, since it takes into account the reversible work spent in the solvent polarization process.

We here report only the final expressions for the Fock matrix to be used in the self-consistent field approximation (SCF) of the electronic wave function,

$$
f_{p q}=f_{p q}^{\mathrm{vac}}+\left(\sigma, \varphi_{p q}\right)_{\partial C}
$$

where the usual vacuum terms are augmented by a solvent term,

$$
\left(\sigma, \varphi_{p q}\right)_{\partial C}=\int_{\partial C} \mathrm{~d} \boldsymbol{s} \sigma(\boldsymbol{s}) \varphi_{p q}(\boldsymbol{s}),
$$

and detailed derivations can be found in the existing literature. ${ }^{18,70}$ The Fock matrix expression is in the molecular orbital (MO) basis. The usual notation for the indices is adopted: $i, j, k, \ldots$ for occupied orbitals and $a, b, c, \ldots$ for virtual orbitals, while $p, q, r, \ldots$ for general orbitals. The vacuum-like term is given as

$$
f_{p q}^{\mathrm{vac}}=h_{p q}+\sum_{j}\left(g_{p q j j}-\gamma g_{p j j q}\right)+v_{\mathrm{xc} ; p q}
$$

and also describes the case of Kohn-Sham DFT, possibly using hybrid functionals. ${ }^{71}$ The integrals $\varphi_{p q}(s)$ are called charge-attraction integrals,

$$
\varphi_{p q}(\boldsymbol{s})=\int \mathrm{d} \boldsymbol{r} \frac{-\chi_{p}^{*}(\boldsymbol{r}) \chi_{q}(\boldsymbol{r})}{|\boldsymbol{r}-\boldsymbol{s}|} .
$$

\section{IMPLEMENTATION}

The calculation of the Green's function for a diffuse interface requires the numerical resolution of Eq. (16), a second order ordinary differential equation. In Eq. (17), we assumed a power form of the solutions to Eq. (16) in the asymptotic regime. This is numerically impractical: it requires the evaluation of the power function with, potentially, large exponents and might incur into arithmetic overflows. It would be possible to use an arbitrary precision representation of floating-point numbers, but it is more convenient to perform a transformation to an equivalent form that is better behaved numerically,

$$
R_{\ell}=\exp \left(\rho_{\ell}\right)
$$

such that

$$
\frac{\mathrm{d}^{2} \rho_{\ell}}{\mathrm{d} r^{2}}+\left(\frac{\mathrm{d} \rho_{\ell}}{\mathrm{d} r}\right)\left(\frac{\mathrm{d} \rho_{\ell}}{\mathrm{d} r}+\frac{2}{r}+\lambda_{\varepsilon}(r)\right)-\frac{\ell(\ell+1)}{r^{2}}=0
$$

which is a nonlinear second-order ODE. The two transformed independent solutions will be denoted by $\zeta_{\ell}$ and $\omega_{\ell}$. Their asymptotic behaviour is accordingly,

$$
\zeta_{\ell}=\ell \ln (r) \quad \omega_{\ell}=-(\ell+1) \ln (r)
$$

as can be seen from Eq. (17). The exponential transformation is particularly convenient, since it is differentiable and invertible on the entire problem domain $[0,+\infty)$. The Green's function in Eq. (31) is then given as

$$
G\left(\boldsymbol{r}, \boldsymbol{r}^{\prime}\right)=\sum_{\ell=0}^{L} \bar{G}_{\ell}\left(\boldsymbol{r}, \boldsymbol{r}^{\prime}\right)=\left\{\begin{array}{l}
\sum_{\ell=0}^{L} \frac{2 \ell+1}{\left.r^{\prime 2} \varepsilon\right|_{r^{\prime}}} \frac{\exp \left(\left.\zeta_{\ell}\right|_{r}-\left.\zeta_{\ell}\right|_{r^{\prime}}\right)}{\frac{\left.\mathrm{d} \zeta_{\ell}\right|_{\mathrm{d}}-\left.\frac{\mathrm{d} \omega_{\ell}}{\mathrm{d} r}\right|_{r^{\prime}}}{\mathrm{d}} P_{\ell}(\cos \gamma), \text { for } r<r^{\prime},} \\
\sum_{\ell=0}^{L} \frac{2 \ell+1}{\left.r^{\prime 2} \varepsilon\right|_{r^{\prime}}} \frac{\exp \left(\left.\omega_{\ell}\right|_{r}-\left.\omega_{\ell}\right|_{r^{\prime}}\right)}{\left.\frac{\mathrm{d} \zeta_{\ell}}{\mathrm{d} r}\right|_{r^{\prime}}-\left.\frac{\mathrm{d} \omega_{\ell}}{\mathrm{d} r}\right|_{r^{\prime}}} P_{\ell}(\cos \gamma), \text { for } r>r^{\prime},
\end{array}\right.
$$

and the angular momentum components summation was truncated to $L$ terms, with $L$ arbitrarily large. $\bar{G}_{\ell}\left(\boldsymbol{r}, \boldsymbol{r}^{\prime}\right)$ is the $\ell$ th angular momentum component calculated in the transformed representation. For the calculation of the separation coefficient one has

$$
C\left(\boldsymbol{r}, \boldsymbol{r}^{\prime}\right)=\left\{\begin{array}{l}
\frac{1}{r^{\prime}}\left(\frac{r}{r^{\prime}}\right)^{2 L} \frac{P_{2 L}(\cos \gamma)}{\bar{G}_{2 L}\left(\boldsymbol{r}, \boldsymbol{r}^{\prime}\right)}, \text { for } r<r^{\prime}, \\
\frac{1}{r}\left(\frac{r^{\prime}}{r}\right)^{2 L} \frac{P_{2 L}(\cos \gamma)}{\bar{G}_{2 L}\left(\boldsymbol{r}, \boldsymbol{r}^{\prime}\right)}, \text { for } r>r^{\prime},
\end{array}\right.
$$

where the limit for infinite $\ell$ was replaced by the value for $\ell=2 L$

As apparent from Section II, the same type of radial ODE occurs regardless of the shape of the dielectric profile describing the diffuse transition layer. It is thus convenient to provide an implementation of the diffuse dielectric layer Green's function which is generic with respect to the functional form of the permittivity profile. The generic ODE integrators library Boost.Odeint ${ }^{72,73}$ is currently used. In particular, the adaptive Bulirsch-Stoer algorithm ${ }^{74}$ is selected among the many steppers provided by the library. 
The nonlinear second-order ODE in Eq. (56) is recast as a system of coupled nonlinear first-order ODEs,

$$
\left\{\begin{array}{l}
\frac{\mathrm{d} \rho_{\ell}}{\mathrm{d} r}=\sigma_{\ell} \\
\frac{\mathrm{d} \sigma_{\ell}}{\mathrm{d} r}=-\sigma_{\ell}\left(\sigma_{\ell}+\frac{2}{r}+\lambda_{\varepsilon}(r)\right)+\frac{l(l+1)}{r^{2}}
\end{array}\right.
$$

Appropriate boundary conditions have to be enforced to ensure the correct solution of the homogeneous problem Eq. (16). Given that ODE integration is carried out on a finite interval $\left[r_{0}, r_{\infty}\right]$ the boundary conditions to impose are naturally derived from Eq. (17):

$$
\left\{\begin{array}{l}
\left.\zeta_{\ell}\right|_{r_{0}}=\ell \ln \left(r_{0}\right) \\
\left.\frac{\mathrm{d} \zeta_{\ell}}{\mathrm{d} r}\right|_{r_{0}}=\frac{\ell}{r_{0}}
\end{array}, \quad\left\{\begin{array}{l}
\left.\omega_{\ell}\right|_{r_{\infty}}=-(\ell+1) \ln \left(r_{\infty}\right) \\
\left.\frac{\mathrm{d} \omega_{\ell}}{\mathrm{d} r}\right|_{r_{\infty}}=-\frac{\ell+1}{r_{\infty}}
\end{array} .\right.\right.
$$

The integrator stepping function is set to perform a forward, i. e., from $r_{0}$ until $r_{\infty}$, integration when $\zeta_{\ell}$-type solutions are requested. Backward steps are performed, i. e., from $r_{\infty}$ until $r_{0}$, when $\omega_{\ell}$-type solutions are requested. The ODE system is solved for a dielectric sphere centered at the origin of the coordinate system. The correct translation is applied when sampling the Green's function for arbitrary pairs of points-r, $\boldsymbol{r}^{\prime}$-on the cavity: for a sphere centered in $\boldsymbol{t}$, we evaluate $G\left(\boldsymbol{r}+\boldsymbol{t}, \boldsymbol{r}^{\prime}+\boldsymbol{t}\right)$. For the sampling, interpolations between points on the radial grids have to be performed. In the current implementation, a cubic spline interpolation is performed using the facility provided by the Eigen library. ${ }^{75}$ The evaluation of the Green's function directional derivative, required by the collocation formulas for the $\mathcal{D}_{\mathrm{e}}$ operator, is done numerically using a three-point stencil.

The implementation has been included in our PCMSolver library ${ }^{76}$ described also in Refs. 77 and 78. The Green's function here described is implemented as an extension to the Green's function module of the library. The calculation can be set up by providing a suitable permittivity profile, the sphere center $\boldsymbol{t}$, and the value of $L$ in Eqs. (58) and (59). Following Ref. 39, the code assumes the permittivity profiles to be of the form

$$
\varepsilon(r)=\frac{\varepsilon_{1}+\varepsilon_{2}}{2}+\frac{\varepsilon_{2}-\varepsilon_{1}}{2} \Sigma\left(\frac{r-c}{w}\right),
$$

where $\varepsilon_{1}, \varepsilon_{2}$ are the bulk permittivities inside and outside the sphere, respectively. The center of the interface- $c$-is defined as the center of the transition layer, its value corresponding to the radius of the dielectric sphere. $w$ is the width of the transition layer. To keep again consistency with Ref. 39, the width parameter appearing in Eq. (62) is set to be $w=\frac{\tilde{w}}{6}$, where $\tilde{w}$ is the width given by the user in the input. Our notation is slightly different from the one adopted by Frediani et $a l .{ }^{39}$ In particular, the $D$ parameter in their Eq. (29) corresponds to our $w$ in Eq. (62), thus their $W$ is equivalent to our $\tilde{w}$. Finally, $\Sigma$ is a sigmoidal function. The currently supported profile functions are the hyperbolic tangent, as in Ref. 39, and the error function. Figure 1 shows the permittivity profiles and corresponding Onsager factors $f(\varepsilon)=\frac{\varepsilon(r)-1}{\varepsilon(r)}$ with different widths $\tilde{w}$ with a hyperbolic tangent sigmoidal function. More details on the permittivity profiles

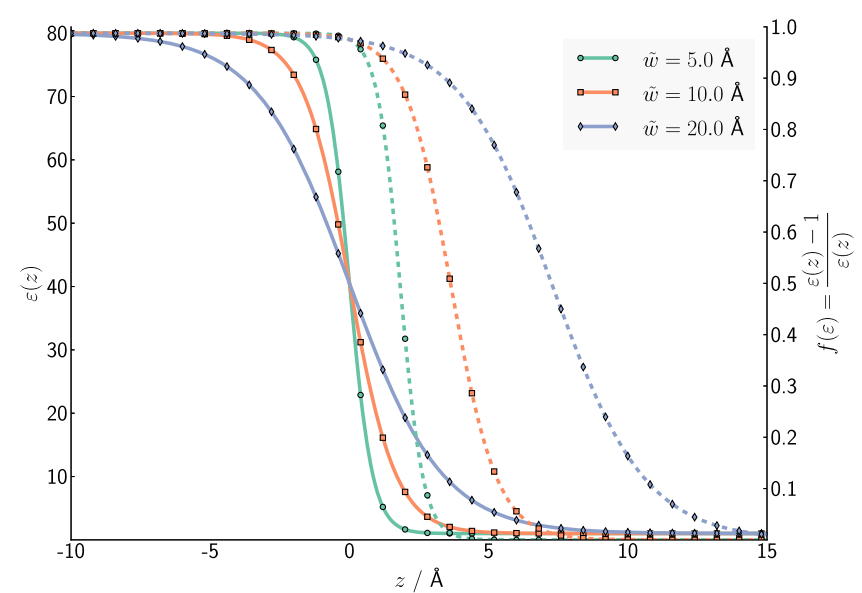

FIG. 1. Permittivity profiles (solid lines) and Onsager factors $f(\varepsilon)=\frac{\varepsilon-1}{\varepsilon}$ (dashed lines) as a function of the distance from the interface center $z$. $\varepsilon(r)$ is parametrized in terms of Eq. (62) with $\Sigma(x)=\tanh (x)$. A spherical droplet with radius $c=10.0 \AA$ and centered at the origin is assumed. The bulk permittivities are set to $\varepsilon_{1}=80.0$ and $\varepsilon_{2}=1.0$. The width $\tilde{w}$ is varied among $5.0 \AA$ (green), $10.0 \AA$ (orange) and $20.0 \AA$ (blue).

are given in Appendix. The integration domain is kept fixed: $r_{0}=0.5 a_{0}$, and $r_{\infty}=R+200.0 a_{0}$, where $R$ is the sphere radius.

The recently published interface with the LSDALTON code $^{79}$ has been used in the quantum mechanical calculations. ${ }^{78}$

\section{NUMERICAL TESTS}

\section{A. Computational details}

All the calculations, including geometry optimizations and calculation of solvation energies, were carried out with B3LYP functional ${ }^{80,81}$ and basis set of aug-cc-pVDZ quality. ${ }^{82}$ The geometries were optimized in water using the IEF-PCM ${ }^{58,83}$ as implemented in the Gaussian03 program package. ${ }^{84}$ Diffuse interface calculations were carried out using the LSDALTON program package. ${ }^{79}$ Geometries were kept fixed for the interface calculations.

Unless otherwise specified, all the molecules were oriented such that the principal molecular symmetry axis is collinear with the normal vector to the interface. For the three investigated molecules (Fig. 2), the aforementioned groups, pointing towards the water droplet, are as follows: oxygen for acetone, $\mathrm{NO}_{2}$ group for $p$-nitroaniline (PNA) and the carboxylic group for L0 dye from Ref. 50. Additionally, for acetone, an orientation in which the dipole moment of the molecule is perpendicular to the interface normal vector is considered. In the analysis of the results, we will adopt the following convention: $r$ is the distance from the origin, $R_{0}$ is the radius of the medium sphere, and $z=r-R_{0}$ is used to indicate the distance from the interface.

Molecular cavities were created from interlocking, atomcentered spheres. The following set of radii was used: $1.44 \AA$ for hydrogens capable of hydrogen bonding, $1.824 \AA$ for oxygen, $1.92 \AA$ for nitrogen, $2.34 \AA$ for bromide, $1.226 \AA$ for lithium cation, and $2.16 \AA$ for carbon. For $\mathrm{CH}, \mathrm{CH}_{2}$, and $\mathrm{CH}_{3}$ 


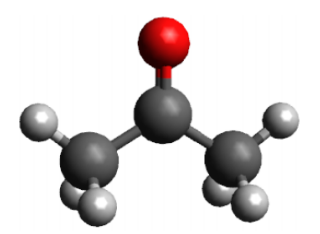

(a)

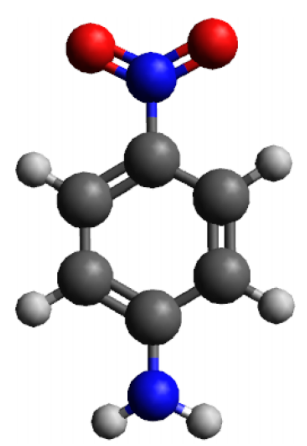

(b)

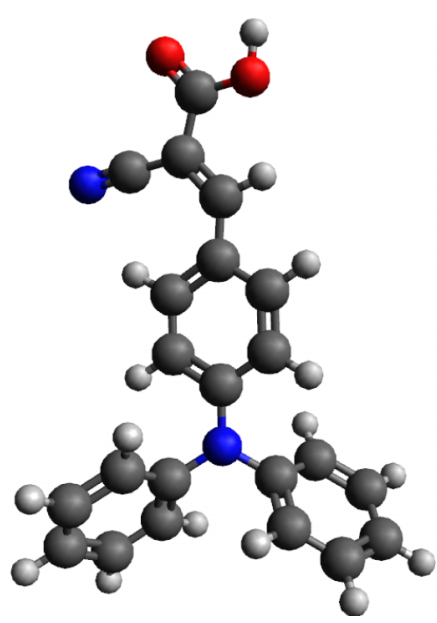

(c)
FIG. 2. Geometry of the molecules used in this paper. (a) Acetone. (b) PNA. (c) L0 dye. groups radii equal to $2.28 \AA, 2.4 \AA$, and $2.52 \AA$ were used, respectively.

\section{B. Transport across the spherical diffuse interface: Effect of the interface width}

We consider here how the solvation energy profile through the interface changes when the interface width is varied between 5 and $20 \AA$. The radius of the droplet is kept fixed to $R_{0}=20 \AA$.

In Fig. 3, we report the electrostatic solvation profile for $\mathrm{Li}^{+}$and $\mathrm{Br}^{-}$crossing the surface of a water droplet. The solvation energies in bulk are $-134 \mathrm{kcal} / \mathrm{mol}$ and $-65 \mathrm{kcal} / \mathrm{mol}$ for $\mathrm{Li}^{+}$and $\mathrm{Br}^{-}$, respectively. For both ions, the solvation energy changes smoothly from the bulk value (left) to zero (right) in gas-phase. A wider profile yields a smoother transition. Bromide values are smoother than lithium values, due to the size of the ion. We also note that, when the ions are located at the center of the interface $(r=20 \AA)$, the solvation energy is very close to the bulk value, which is the saturation effect caused by the Onsager factor $(\varepsilon(z)-1) / \varepsilon(z)$ being close to its bulk value (see Fig. 1) In other words, from the electrostatic point of view, the interfacial area is rather outside the droplet than inside.

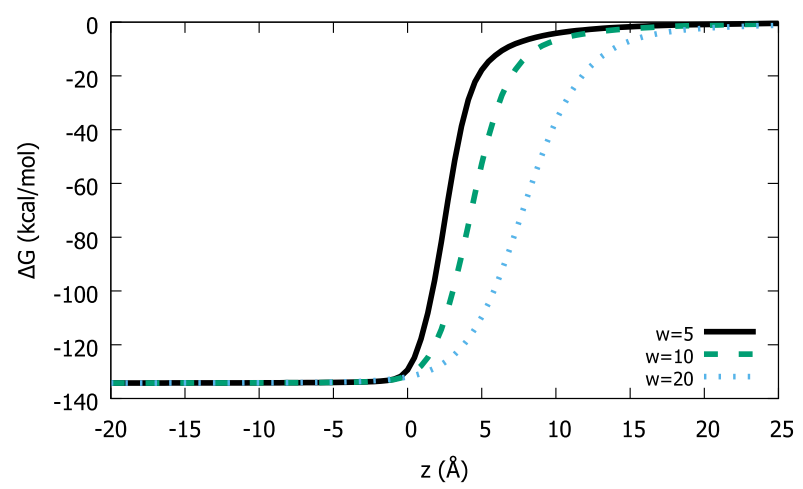

(a)
In Fig. 4, we report the solvation energy profile for acetone with the dipole moment parallel (Fig. 4(a)) and perpendicular (Fig. 4(b)) to the interface. The same saturation effect observed for ions can be seen here. Moreover, for the perpendicular case and the sharpest interface (Fig. 4(b), $\tilde{w}=5 \AA$ ) the profile is not as smooth as the others. This feature is the result of a polarization effect arising when the two extremities of the molecule experience a widely different polar environment with the oxygen atom feeling almost full solvation and the methyl groups still in a vacuum-like environment. For wider profiles or for the parallel orientation such a feature disappears, because the molecule experiences a more uniform environment.

In Fig. 5 we report the electrostatic solvation profiles of PNA and L0 crossing the interface for different widths of the profile. Similarly to acetone, the sharpest interface $(\tilde{w}=5 \AA)$ yields a highly inhomogeneous solvation which is polarizing the substrate and generating the features along the profile. Such features tend to disappear for larger interfacial widths. The size of the molecules plays also a role, widening the profiles: for PNA, a significant saturation effect can still be observed (bulk solvation is basically reached at $z=-5 \AA$ for all widths) whereas for $\mathrm{L} 0$, the saturation effects are much less important (bulk solvation reached at $z=-10 \AA$, which is the end of the interfacial region also for $\tilde{w}=20 \AA$ ).

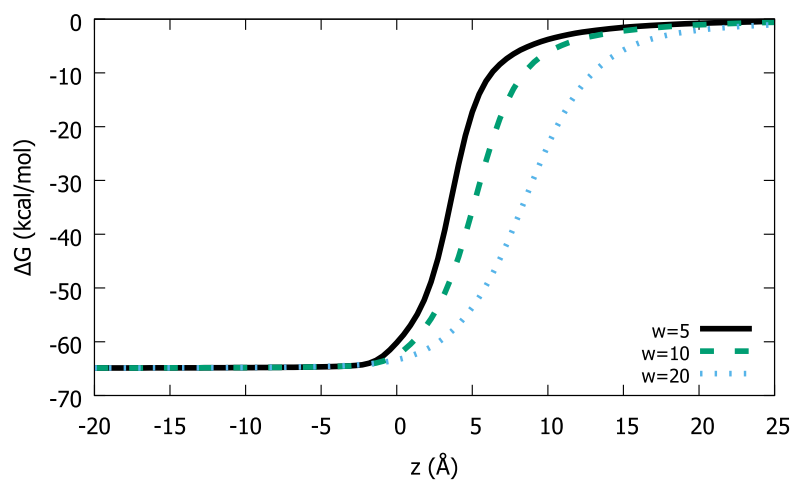

(b)

FIG. 3. Electrostatic energy profile for the water-vapor transfer of $\mathrm{Li}^{+}$and $\mathrm{Br}^{-}$. A droplet with $R_{0}=20 \AA$, and interfacial width $\tilde{w}$ as denoted in the legend (in $\AA$ ) is considered. (a) $\mathrm{Li}^{+}$. (b) $\mathrm{Br}^{-}$. 


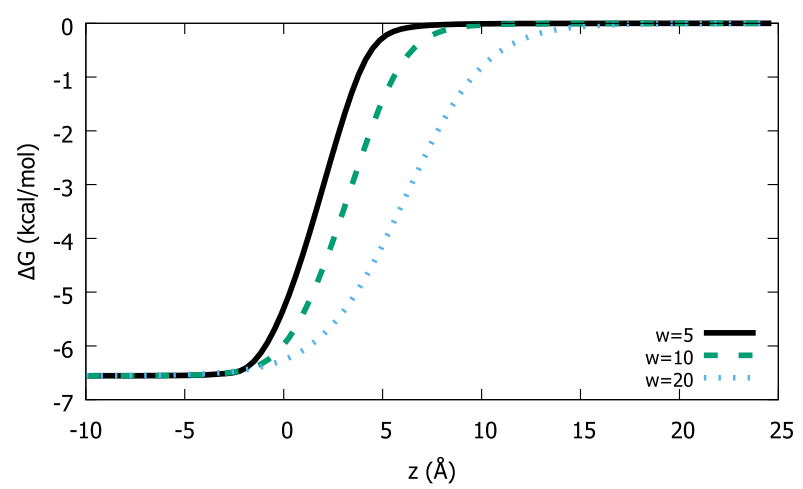

(a)

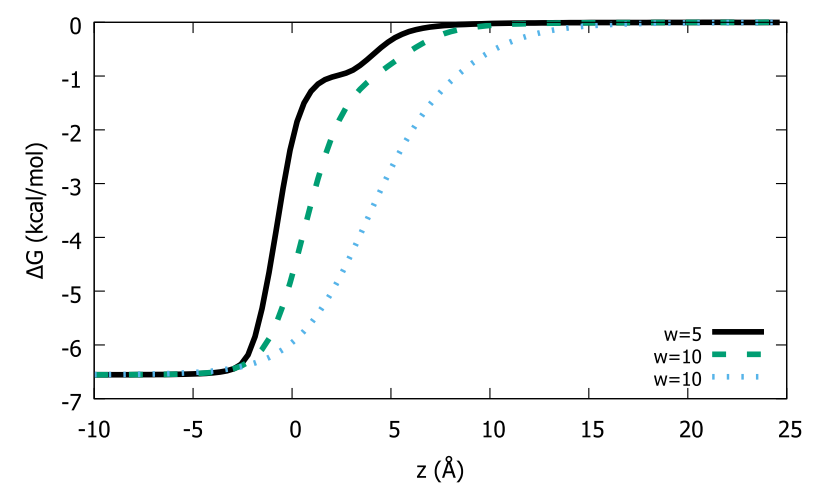

(b)

FIG. 4. Electrostatic energy profile for the water-vapor transfer of acetone molecule with dipole moment parallel and perpendicular to the interface. A droplet with $R_{0}=20 \AA$, and interfacial width $\tilde{w}$ as denoted in the legend (in $\AA$ ) is considered. (a) Parallel. (b) Perpendicular.

\section{Transport across the spherical diffuse interface: Curvature effect}

In order to analyze the curvature effect, we have considered spherical interfaces with a width of $\tilde{w}=5 \AA$ and increasing radius $\left(R_{0}=5,10,20,40\right.$, and $\left.80 \AA\right)$. We have moreover considered three cases: a lithium ion across the interface of a water droplet in vacuum, a lithium ion across the interface of an apolar solvent $(\varepsilon=2)$ in water, and acetone across the surface of a water droplet (perpendicular orientation). In order to highlight the curvature effects, we have reported both the total solvation energy $E_{R_{0}}(r)$ for each value of $R_{0}$ (see Fig. 6) and the difference,

$$
\Delta E_{R_{0}}(z)=E_{R_{0}}\left(z+R_{0}\right)-E_{R_{0}=80}(z+80),
$$

as a function of the distance to the interface (see Fig. 7).

The main feature to highlight in Fig. 7(a) is that there is basically no curvature effect on bulk solvation. In other words, a molecule situated at the center of the droplet, provided that the permittivity has reached its bulk value at the molecular cavity surface, will experience the same dielectric response as a molecule situated in bulk. For a spherical ion at the center of a spherical droplet, this can very simply be understood in terms of the Gauss' law for dielectric materials. Assuming that at the cavity boundary $\varepsilon(r) \simeq \varepsilon$, the Gauss' law dictates that, for a unit charge, the apparent charge at the cavity $(1-\varepsilon) / \varepsilon$, which is the same value as for bulk solvation. Due to the spherical symmetry of the problem, this will yield the same solvation energy as for a charge in bulk. Although spherical symmetry no longer applies for ions away from the origin or for molecules, this simple argument shows why the asymptotic value is virtually identical to the bulk value: the largest deviation was observed for L0 with $14 \mu E_{\mathrm{h}}$ hartree in the solvation energy (less than $10^{-3} \mathrm{kcal} / \mathrm{mol}$ ). For the water droplet, basically no change in the solvation energy is observed until the interfacial area is reached and after a sudden jump across the interface, the solvation energy gradually approaches zero. The behavior before and after the interface is connected to the high permittivity of water: inside the droplet, high dielectric screening makes the droplet almost indistinguishable from bulk water, until the interface is reached. Outside the droplet, the large image potential is still felt at medium-long range. For an oil droplet in water, the behavior is opposite: inside the droplet, the solvation energy changes when the substrate is displaced, but outside the droplet, the image effect is negligible. The profiles for acetone show that the irregular profile, described in Section IV B, is a combined effect of profile width and curvature: for very small droplets (5 and $10 \AA$ ), it is enhanced, whereas for large ones (40 and $80 \AA$ ), it is less noticeable.

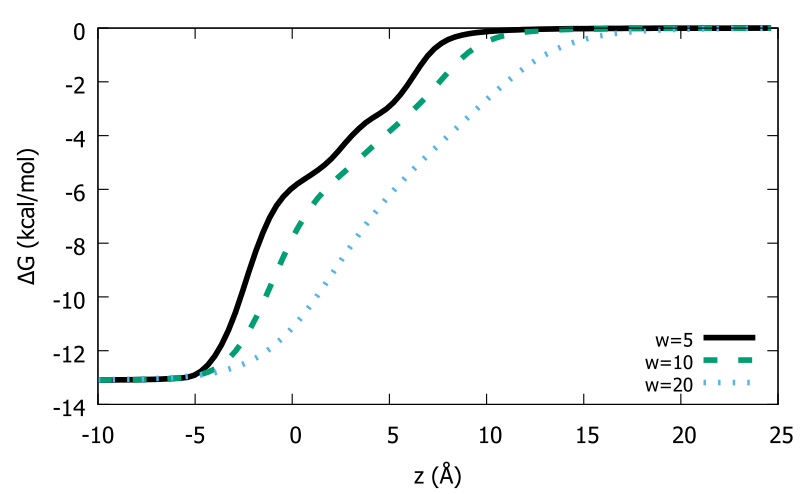

(a)

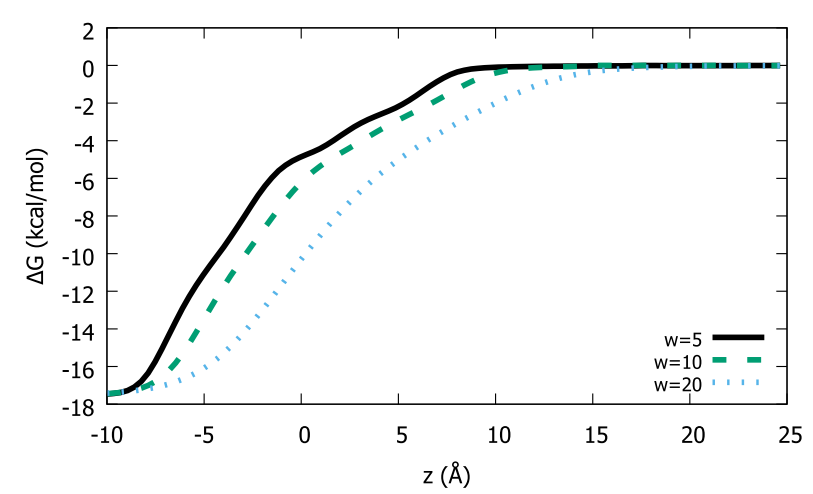

(b)

FIG. 5. Electrostatic energy profile for the water-vapor transfer of PNA molecule and L0 dye from Ref. 50. A droplet with $R_{0}=20 \AA$, and interfacial width $\tilde{w}$ as denoted in the legend (in $\AA$ ) is considered. (a) PNA. (b) L0 dye from Ref. 50. 


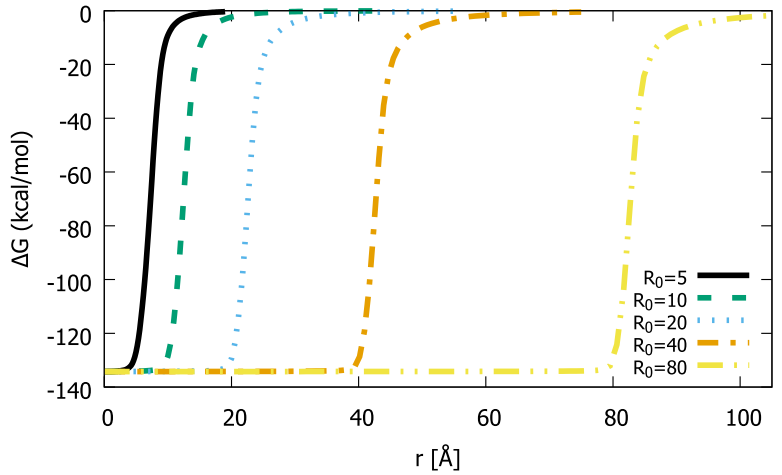

(a)

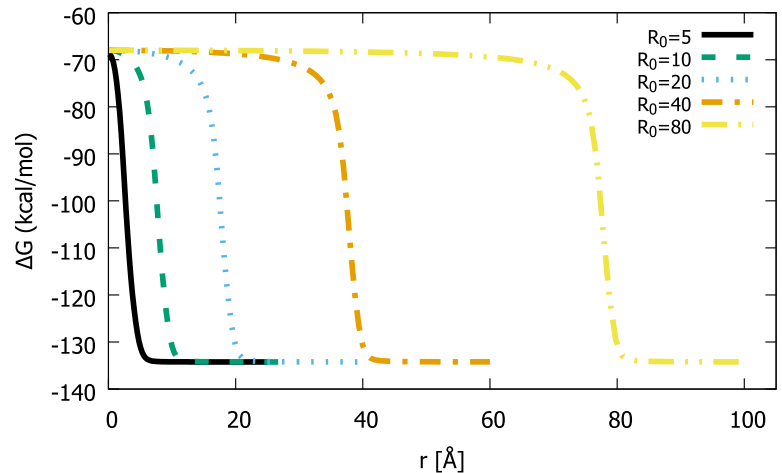

(b)

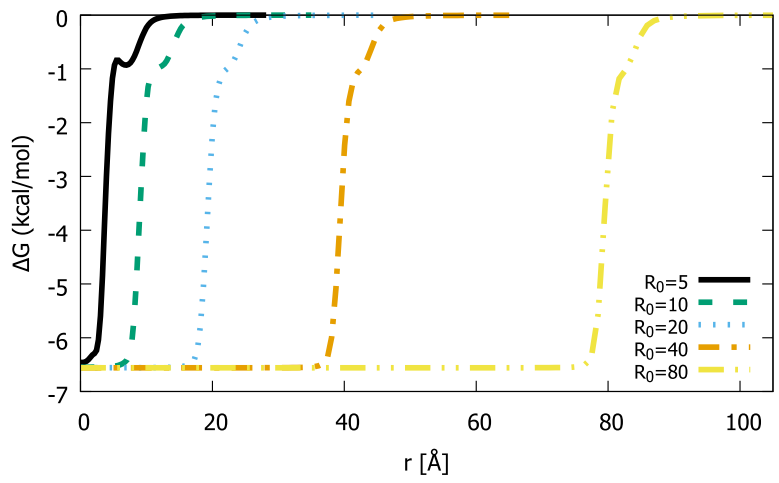

(c)

FIG. 6. Electrostatic energy of solvation profiles for acetone with dipole moment perpendicular to the interface for varying values of $R_{0}$. Systems examined are water droplet in air and oil droplet in water. Interface width of $5 \AA$ was used. (a) $\mathrm{Li}^{+}$, water droplet in air. (b) $\mathrm{Li}^{+}$, oil droplet in water. (c) Acetone, water droplet in air.

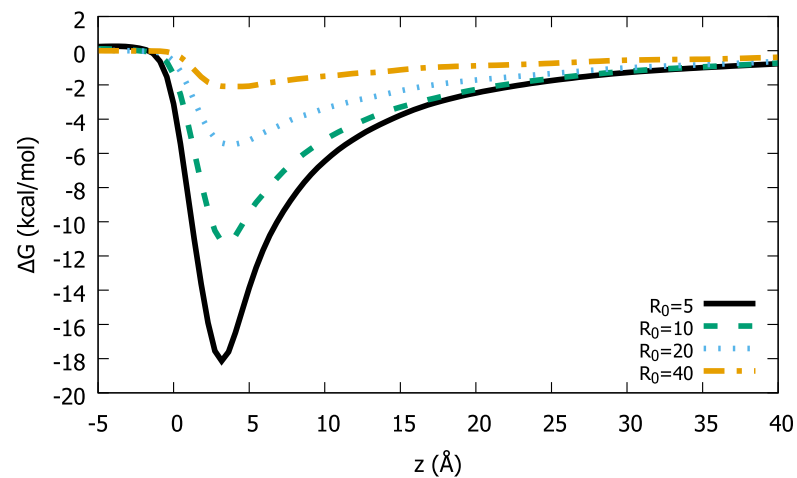

(a)

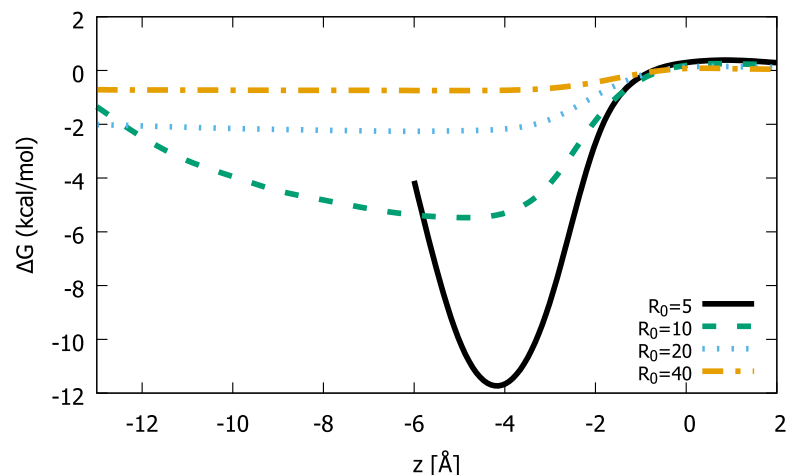

(b)

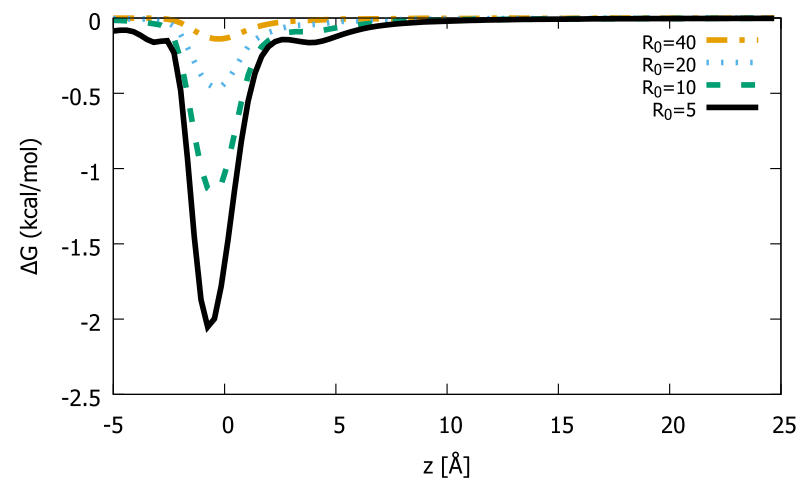

(c)

FIG. 7. Differences in solvation energy for varying values of $R_{0}$ with respect to $R_{0}=80 \AA$. Systems examined are: water droplet in air and oil droplet in water. Interface width of $5 \AA$ was used. (a) $\mathrm{Li}^{+}$, water droplet in air. (b) $\mathrm{Li}^{+}$, oil droplet in water. (c) Acetone, water droplet in air. 


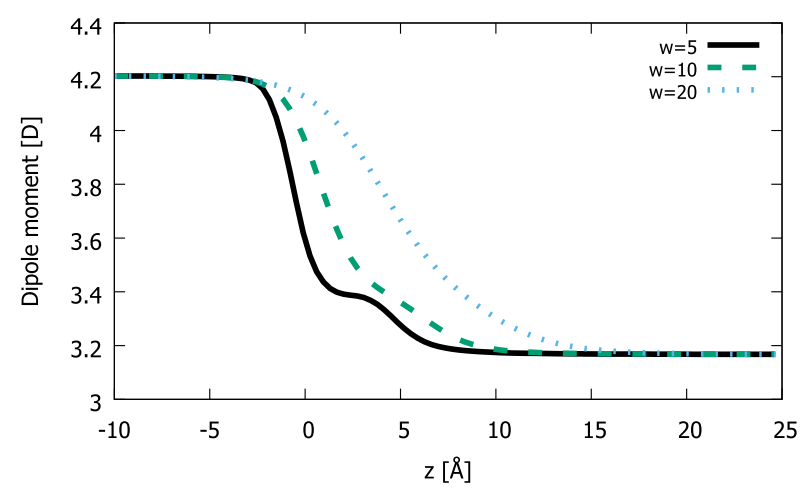

(a)

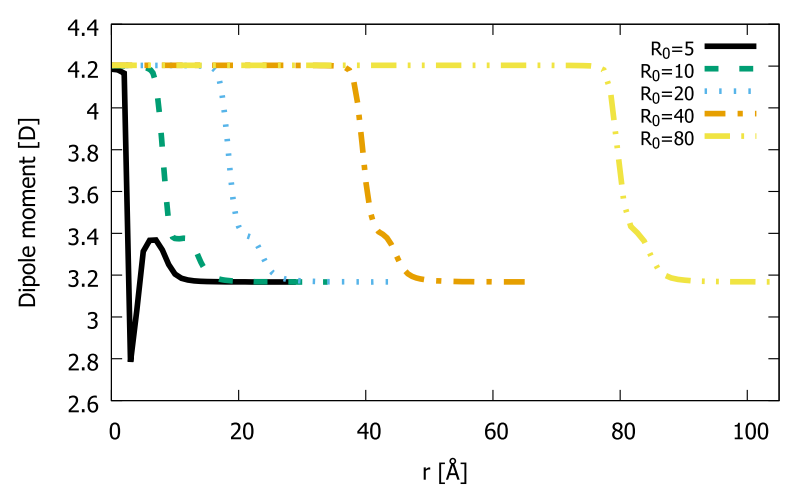

(b)

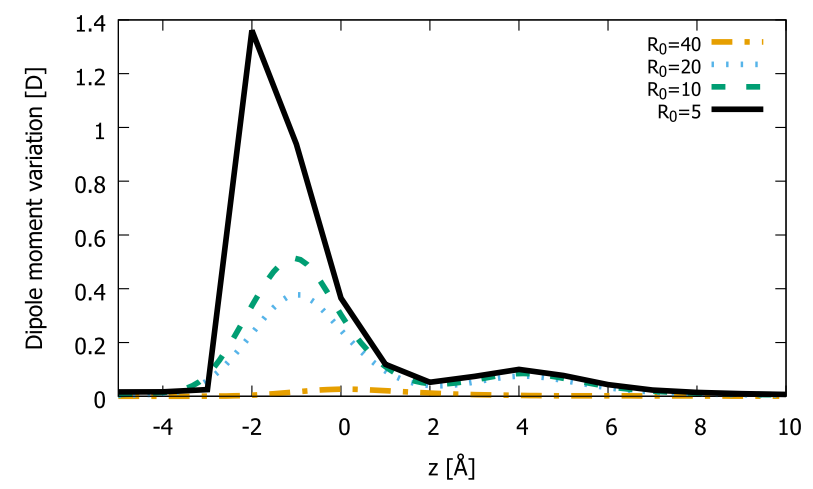

(c)

FIG. 8. Dipole moment profiles for acetone (with dipole moment perpendicular to the interface) transfer at water droplet in air, for varying interface widths, values of $R_{0}$ and the curvature effect on dipole moment, respectively. (a) Width effect. (b) Radius effect. (c) Curvature effect.

In order to investigate the curvature effect around the interfacial area, for each radius $R_{0}$, we have considered $\Delta E_{R_{0}}$ as defined in Eq. (63), such that for each profile, the interface is located at the origin. We have chosen to take $R_{0}=80 \AA$ as the reference value, instead of a planar interface. The implementations for the planar and spherical diffuse environments are different and we wanted to avoid any implementation bias in the comparison. The plots in Fig. 7 illustrate such an effect for a selection of the system investigated here. The curvature effect for the $\mathrm{Li}^{+}$ion at the surface of the water droplet (see Fig. 7(a)) can be as large as $-18 \mathrm{kcal} / \mathrm{mol}$ for the smallest radius and it occurs before the ion crosses the surface (positive $z$ ). On the contrary, for the oil droplet in water (see Fig. 7(b)), the curvature effect is pronounced after the ion has crossed the interface. For the acetone molecule at the water droplet (see Fig. 7(c)) the effect is instead centered at the interface and can be as large as $-2 \mathrm{kcal} / \mathrm{mol}$. Although the magnitude of the variations is in absolute value much smaller than what observed for lithium, they are in relative terms much larger, if bulk solvation is taken as a reference (30\% vs. $13 \%)$. In conclusion we see a substantial curvature effect in the solvation energy, both for charged and dipolar substrates. For charged substrates the effect is prominent on the low-polarity side of the interface, whereas for dipolar substrate, it is roughly centered at the interface. The first observation correlates well with the saturation effect, and consequent screening in the dielectric response for solvents with high polarity: when the ion is in water, the effective charge seen by the interface is much smaller and hence the image-like contribution is small. For a dipolar substrate like acetone, there is also an indirect effect, due to the dipole moment enhancement at the interface, which appears once the oxygen atom has crossed the interface (see Fig. 8, and in particular Fig. 8(c)). As a result the curvature effect appears centered at the interface.

\section{SUMMARY}

We have presented the first implementation of a diffuse dielectric interface in spherical symmetry for the Integral Equation Formalism PCM. We have shown how the necessary Green's function can be obtained by solving numerically the radial differential equation for each component of the angular momentum expansion. To achieve a numerically robust solution, special care is needed to treat the Coulomb singularity, which is removed from each component of the multipolar expansion and included in its analytical form instead. This allows one to significantly limit the expansion, which at short distances would otherwise be dominated by large $\ell$-values to describe the Coulomb singularity.

We have performed a few numerical tests to investigate the effect of the interface width $\tilde{w}$, and the droplet radius $R_{0}$. For monoatomic ions, the solvation energy profile correlates very well with the Onsager factor $(\varepsilon-1) / \varepsilon$, showing that most of the interfacial effect is in this case local. However the curvature effect is also important: on the low-polarity side of the interface, a more pronounced curvature (smaller $R_{0}$ ) 
acts by anticipating solvation to larger distances from the interface. For larger molecular substrates, the outcome is also largely dependent on the structure and orientation of the molecule.

Although we have limited this study to the case of nanodroplets, with a simple sigmoidal shape for the permittivity (see Appendix), our approach is general and can be used for continuously differentiable radial profiles that exhibit a constant permittivity in the limit of large $r$. The present work will open the way for several interesting applications: solvation effects from nanodroplets on molecular properties, the modeling of coated nanoparticles and membranes and the combination of this model with a Polarizable Embedding (PE) scheme $^{85}$ to include also specific intermolecular interactions.

\section{ACKNOWLEDGMENTS}

The authors would like to thank Dr. Stefano Corni (Center S3, CNR Institute of Nanoscience, Modena) for insightful discussions and suggestions on the separation of the Coulomb singularity. The computational resources for this project have been provided by the NOTUR high performance computing program (Grant No. NN4654K). This work has been supported by the Research Council of Norway through a Centre of Excellence Grant (Grant No. 179568/V30). L.F., K.M., and V.W. also acknowledge financial support by the Troms $\varnothing$ Research Foundation (SURFINT grant). H.C. acknowledges the Jiangsu Specially Appointed Professor Program (No. R2013T08).

\section{APPENDIX: DIELECTRIC PERMITTIVITY PROFILES}

As explained in Sec. III, the code assumes the permittivity profiles to be of the form

$$
\varepsilon(r)=\frac{\varepsilon_{1}+\varepsilon_{2}}{2}+\frac{\varepsilon_{2}-\varepsilon_{1}}{2} \Sigma\left(\frac{r-c}{w}\right),
$$

where $\varepsilon_{1}, \varepsilon_{2}$ are the bulk permittivities inside and outside the sphere, respectively. The center of the interface is defined as

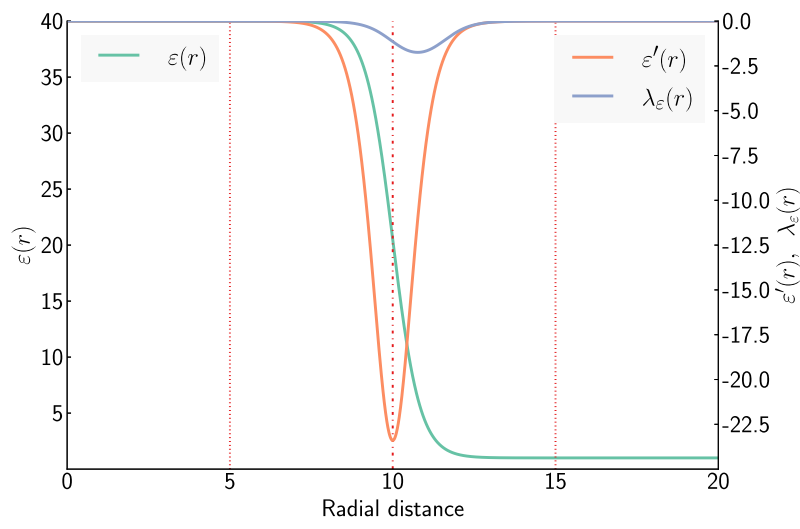

(a) the center of the transition layer, its value corresponding to the radius of the dielectric sphere. $w$ is the width of the transition layer. Once again, to keep consistency with Ref. 39, the width parameter appearing in Eq. (62) is set to be $w=\frac{\tilde{w}}{6}$, where $\tilde{w}$ is the width given by the user in the input.

Some further remarks are needed on the functional form of $\Sigma$ and the meaning of its parameters. Eq. (14) is the ODE governing the radial part of the Green's function,

$$
\begin{aligned}
\frac{\mathrm{d}^{2} R_{\ell}}{\mathrm{d} r^{2}} & +\left(\frac{2}{r}+\lambda_{\varepsilon}(r)\right) \frac{\mathrm{d} R_{\ell}}{\mathrm{d} r}-\frac{\ell(\ell+1)}{r^{2}} R_{\ell} \\
& =-\frac{4 \pi}{r^{2}} \frac{1}{\varepsilon(r)} \delta\left(r-r^{\prime}\right),
\end{aligned}
$$

which features both the inverse of the permittivity profile and the weighted dielectric distribution Eq. (9),

$$
\lambda_{\varepsilon}(r)=\frac{\mathrm{d} \varepsilon(r)}{\mathrm{d} r} \frac{1}{\varepsilon(r)},
$$

as coefficients. We noted already in Sec. II that this implies $\varepsilon(r)>0$, i. e., the permittivity profile is physically sound if and only if it is a strictly positive function on the whole problem domain. Moreover, given that its first derivative,

$$
\frac{\mathrm{d} \varepsilon(r)}{\mathrm{d} r}=\frac{\varepsilon_{2}-\varepsilon_{1}}{2 w} \frac{\mathrm{d} \Sigma}{\mathrm{d} r}\left(\frac{r-c}{w}\right),
$$

is needed to properly define $\lambda_{\varepsilon}(r)$, we also need to impose that the permittivity profile be, at least, continuously differentiable on the problem domain: $\varepsilon(r) \in C_{[0,+\infty)}^{1}$. All these conditions are satisfied when $\Sigma$ is a sigmoidal function, such as the hyperbolic tangent,

$$
\tanh (x)=\frac{\mathrm{e}^{x}-\mathrm{e}^{-x}}{\mathrm{e}^{x}+\mathrm{e}^{-x}},
$$

or the error function,

$$
\operatorname{erf}(x)=\frac{2}{\sqrt{\pi}} \int_{0}^{x} \mathrm{e}^{-t^{2}} \mathrm{~d} t
$$

which are the profiles currently available in our code. However, a profile for which $w \rightarrow 0$ is not mathematically acceptable,

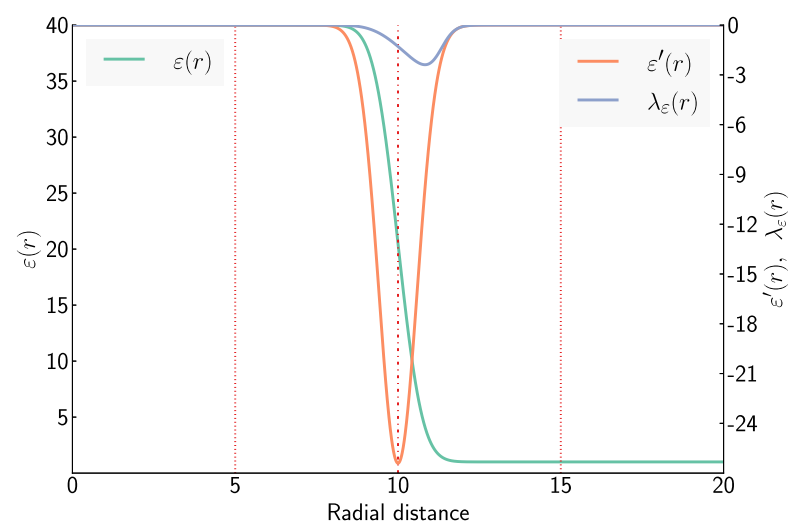

(b)

FIG. 9. Permittivity profiles, their derivatives and the weighted dielectric distribution, in arbitrary units. Profiles are parametrized according to the general Eq. (62). Left panel $\Sigma(x)=\tanh (x)$, right panel $\Sigma(x)=\operatorname{erf}(x)$. In both cases, the profile center is at $c=10$ a.u. and the physical width is set to $\tilde{w}=5$ a.u. The bulk permittivities are set to $\varepsilon_{1}=40.0$ and $\varepsilon_{2}=1.0$ inside and outside, respectively. (a) Permittivity profile parametrized by the hyperbolic tangent. All quantities in arbitrary units. (b) Permittivity profile parametrized by the error function. All quantities in arbitrary units. 


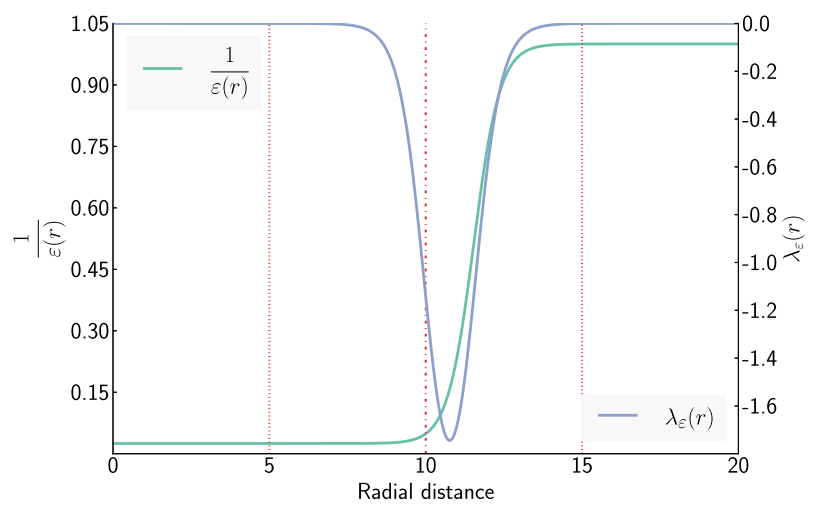

(a)

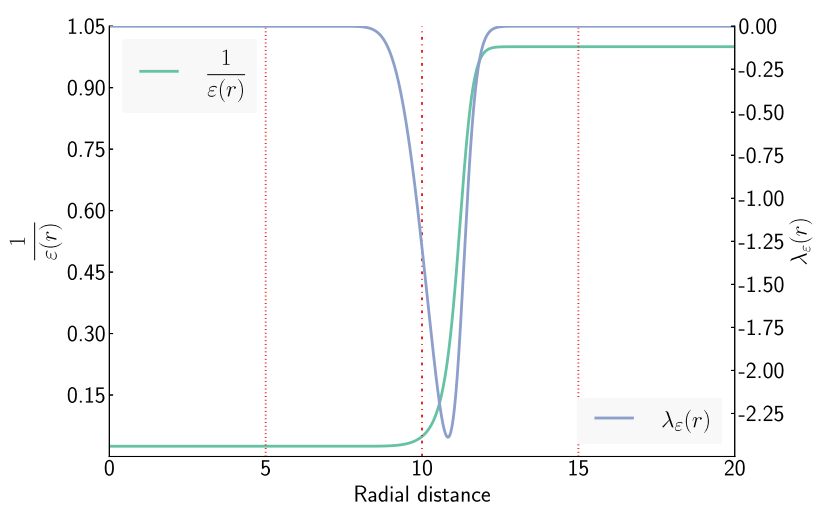

(b)

FIG. 10. Inverse permittivity profiles and the weighted dielectric distribution, in arbitrary units. Profiles are parametrized according to the general Eq. (62). Left panel $\Sigma(x)=\tanh (x)$, right panel $\Sigma(x)=\operatorname{erf}(x)$. In both cases, the profile center is at $c=10$ a.u. and the physical width is set to $\tilde{w}=5$ a.u. The bulk permittivities are set to $\varepsilon_{1}=40.0$ and $\varepsilon_{2}=1.0$ inside and outside, respectively. (a) Permittivity profile parametrized by the hyperbolic tangent. All quantities in arbitrary units. (b) Permittivity profile parametrized by the error function. All quantities in arbitrary units.

since the limit

$$
\lim _{w \rightarrow 0} \frac{\varepsilon_{2}-\varepsilon_{1}}{2 w} \frac{\mathrm{d} \Sigma}{\mathrm{d} r}\left(\frac{r-c}{w}\right)
$$

does not exist. This is true despite the fact that the Green's function for a sharp interface in spherical symmetry is known, see Refs. 49 and 50. Our numerical experiments show that the adaptive Bulirsch-Stoer integrator ${ }^{73}$ can easily handle permittivity profile with $\tilde{w}=6$ (corresponding to $w=1$ ). For lower values of $\tilde{w}$, numerical instabilities are unavoidable and it is then more sensible to use the analytical Green's function for the sharp spherical interface..$^{49,50}$

The hyperbolic tangent profile was suggested by Frediani et al. ${ }^{39}$ based on studies of solvent density profiles. ${ }^{86,87}$ The user-given parameter $\tilde{w}$ is closely related to the physical intuition of a transition layer, i.e., the layer in which the permittivity goes from the inner bulk value to the outer bulk value. This is how Frediani et al. ${ }^{39}$ justified the use of 6 as conversion parameter between the "physical" and the "mathematical" widths. It is possible to offer a simple mathematical explanation for the factor 6 . We will base our analysis on an error function profile, though the same arguments hold for any type of sigmoidal function. The error function can be also seen as the cumulative distribution function associated with a normal probability distribution with mean $\mu=c$ and standard deviation $\sigma=w$. When $\tilde{w}=6 w$, then $99.6 \%$ of the population data will be in the interval $[c-3 w, c+3 w]$. Most importantly in this context, the cumulative distribution function assumes $99.6 \%$ of its left and right asymptotic values at $c-3 w$ and $c+3 w$, respectively. This is shown in Figures 9(a) and 9(b) for a hyperbolic tangent and an error function profile, respectively. Figures 10(a) and 10(b) show the inverse permittivity profile and the weighted dielectric distribution. Notice that the weighted dielectric distribution resembles a distribution function with nonzero moments of order higher than two.
${ }^{4}$ C. E. Kolb, R. A. Cox, J. P. D. Abbatt, M. Ammann, E. J. Davis, D. J. Donaldson, B. C. Garrett, C. George, P. T. Griffiths, D. R. Hanson, M. Kulmala, G. McFiggans, U. Poeschl, I. Riipinen, M. J. Rossi, Y. Rudich, P. E. Wagner, P. M. Winkler, D. R. Worsnop, and C. D. O' Dowd, Atmos. Chem. Phys. 10, 10561 (2010).

${ }^{5}$ The Chemistry of Acid Rain: Sources and Atmospheric Processes, edited by R. W. Johnson and G. E. Gordon, American Chemical Society Symposium Series Vol. 349 (American Chemical Society, Washington, DC, 1987).

${ }^{6}$ A. Rouvière, Y. Sosedova, and M. Ammann, J. Phys. Chem. A 114, 7085 (2010).

${ }^{7}$ K. Eisenthal, Chem. Rev. 106, 1462 (2006).

${ }^{8}$ J. Salafsky and K. Eisenthal, Chem. Phys. Lett. 319, 435 (2000).

${ }^{9}$ Y. Rao, M. Comstock, and K. Eisenthal, J. Phys. Chem. B 110, 1727 (2006).

${ }^{10} \mathrm{~F}$. Vidal and A. Tadjeddine, Rep. Prog. Phys. 68, 1095 (2005).

${ }^{11}$ G. Richmond, Chem. Rev. 102, 2693 (2002).

${ }^{12}$ Even non-centrosymmetric molecules yield no SHG signal in bulk solution due to their random orientation.

${ }^{13}$ R. S. Taylor and B. C. Garrett, J. Phys. Chem. B 103, 844 (1999).

${ }^{14}$ B. Garrett, G. Schenter, and A. Morita, Chem. Rev. 106, 1355 (2006).

${ }^{15}$ S. Miertuš, E. Scrocco, and J. Tomasi, Chem. Phys. 55, 117 (1981).

16J. Tomasi and M. Persico, Chem. Rev. 94, 2027 (1994).

${ }^{17}$ E. Cancès and B. Mennucci, J. Math. Chem. 23, 309 (1998).

${ }^{18}$ J. Tomasi, B. Mennucci, and R. Cammi, Chem. Rev. 105, 2999 (2005).

${ }^{19}$ A. Warshel and M. Levitt, J. Mol. Biol. 103, 227 (1976).

${ }^{20}$ J. L. Gao, Acc. Chem. Res. 29, 298 (1996).

${ }^{21}$ J. Kongsted, A. Osted, K. V. Mikkelsen, and O. Christiansen, Mol. Phys. 100, 1813 (2002).

${ }^{22}$ M. T. C. Martins-Costa, F. F. García-Prieto, and M. F. Ruiz-Lopez, Org. Biomol. Chem. 13, 1673 (2015).

${ }^{23}$ M. T. C. Martins-Costa and M. F. Ruiz-Lopez, J. Phys. Chem. B 117, 12469 (2013).

${ }^{24}$ M. T. C. Martins-Costa, J. M. Anglada, J. S. Francisco, and M. F. RuizLopez, J. Am. Chem. Soc. 134, 11821 (2012)

${ }^{25}$ L. Frediani, C. S. Pomelli, and J. Tomasi, Phys. Chem. Chem. Phys. 2, 4876 (2000).

${ }^{26}$ K. Mozgawa, B. Mennucci, and L. Frediani, J. Phys. Chem. C 118, 4715 (2014).

${ }^{27}$ J. D. Jackson, Classical Electrodynamics (John Wiley \& Sons, 1998).

${ }^{28}$ Y. Zhang, S. E. Feller, B. R. Brooks, and R. W. Pastor, J. Chem. Phys. 103, 10252 (1995).

${ }^{29}$ T.-M. Chang and L. X. Dang, J. Chem. Phys. 104, 6772 (1996).

${ }^{30}$ B. Sokhan and D. J. Tildesley, Mol. Phys. 92, 625 (1997).

${ }^{31}$ F. P. Buff and N. S. Goel, J. Chem. Phys. 51, 4983 (1969).

${ }^{32}$ F. P. Buff and N. S. Goel, J. Chem. Phys. 51, 5363 (1969).

${ }^{33}$ N. S. Goel and F. P. Buff, J. Math. Phys. 11, 508 (1970).

${ }^{34}$ J. R. Clay, N. S. Goel, and F. P. Buff, J. Chem. Phys. 56, 4245 (1972).

${ }^{35}$ F. P. Buff and N. S. Goel, J. Chem. Phys. 56, 2405 (1972).

36J. Perram and M. N. Barber, Mol. Phys. 28, 131 (1974).

${ }^{37}$ F. P. Buff, N. S. Goel, and J. R. Clay, J. Chem. Phys. 63, 1367 (1975).

${ }^{38}$ F. Stern, Phys. Rev. B 17, 5009 (1978).

${ }^{39}$ L. Frediani, R. Cammi, S. Corni, and J. Tomasi, J. Chem. Phys. 120, 3893 (2004). 
${ }^{40}$ C. Xue and S. Deng, Comput. Phys. Commun. 184, 51 (2013)

${ }^{41}$ R. R. Dogonadze and A. A. Kornyshev, J. Chem. Soc., Faraday Trans. 2 70, 1121 (1974).

${ }^{42}$ P. Bopp, A. Kornyshev, and G. Sutmann, Phys. Rev. Lett. 76, 1280 (1996).

${ }^{43}$ A. A. Kornyshev and G. Sutmann, Phys. Rev. Lett. 79, 3435 (1997).

${ }^{44}$ A. Kornyshev and G. Sutmann, J. Mol. Liq. 82, 151 (1999).

${ }^{45}$ M. V. Basilevsky and D. F. Parsons, J. Chem. Phys. 105, 3734 (1996).

${ }^{46}$ M. V. Basilevsky, D. F. Parsons, and M. V. Vener, J. Chem. Phys. 108, 1103 (1998).

${ }^{47}$ M. V. Basilevsky and D. F. Parsons, J. Chem. Phys. 108, 9107 (1998).

${ }^{48}$ M. V. Basilevsky and D. F. Parsons, J. Chem. Phys. 108, 9114 (1998).

${ }^{49}$ R. Messina, J. Chem. Phys. 117, 11062 (2002).

${ }^{50}$ A. Delgado, S. Corni, and G. Goldoni, J. Chem. Phys. 139, 024105 (2013).

${ }^{51}$ S. Deng, J. Electrost. 66, 549 (2008).

${ }^{52}$ S. Deng, J. Electrost. 67, 807 (2009).

${ }^{53}$ S. Deng, Comput. Phys. Commun. 181, 787 (2010).

${ }^{54}$ C. Xue and S. Deng, Phys. Rev. E 81, 016701 (2010).

${ }^{55}$ C. Xue and S. Deng, Phys. Rev. E 83, 056709 (2011).

${ }^{56} \mathrm{~S}$. A. Sauter and C. Schwab, Boundary Element Methods, Springer Series in Computational Mathematics Vol. 39 (Springer, Berlin, Heidelberg, 2011).

${ }^{57}$ G. C. Hsiao and W. L. Wendland, Boundary Integral Equations, Applied Mathematical Sciences Vol. 164 (Springer, Berlin, Heidelberg, 2008).

${ }^{58}$ E. Cancès, B. Mennucci, and J. Tomasi, J. Chem. Phys. 107, 3032 (1997).

${ }^{59}$ J. Vanderlinde, Classical Electromagnetic Theory (Springer, 2005).

${ }^{60}$ G. B. Arfken and H. J. Weber, Mathematical Methods for Physicists (Academic Press, 2005).

${ }^{61}$ E. O. Purisima and S. H. Nilar, J. Comput. Chem. 16, 681 (1995).

${ }^{62}$ C. S. Pomelli, in Continuum Solvation Models in Chemical Physics, edited by B. Mennucci and R. Cammi (John Wiley \& Sons, Ltd., 2007), pp. 49-63.

${ }^{63} \mathrm{~W}$. Hackbusch, Integral Equations: Theory and Numerical Treatment (Birkhaüser, 1995).

${ }^{64}$ E. Silla, J. L. Pascual-Ahuir, J. Tomasi, and R. Bonaccorsi, J. Comput. Chem. 8, 778 (1987).

${ }^{65}$ J. L. Pascual-Ahuir and E. Silla, J. Comput. Chem. 11, 1047 (1990).

${ }^{66}$ C. S. Pomelli and J. Tomasi, Theor. Chem. Acc. 99, 34 (1998).

${ }^{67}$ C. S. Pomelli, J. Tomasi, and R. Cammi, J. Comput. Chem. 22, 1262 (2001).

${ }^{68}$ L. Frediani, R. Cammi, C. S. Pomelli, J. Tomasi, and K. Ruud, J. Comput. Chem. 25, 375 (2004).

${ }^{69}$ J. E. Sanhueza, O. Tapia, W. G. Laidlaw, and M. Trsic, J. Chem. Phys. 70, 3096 (1979).

${ }^{70}$ Continuum Solvation Models in Chemical Physics, edited by B. Mennucci and R. Cammi (John Wiley \& Sons, Ltd., 2007).

${ }^{71}$ T. Helgaker, P. Jørgensen, and J. Olsen, Molecular Electronic-Structure Theory (John Wiley \& Sons, 2000).

${ }^{72}$ Boost C++ Libraries, see http://www.boost.org/.

${ }^{73}$ K. Ahnert and M. Mulansky, AIP Conf. Proc. 1389, 1586 (2011).

${ }^{74}$ F. S. Acton, Numerical Methods that Work (Mathematical Association of America, 1990).

${ }^{75}$ G. Guennebaud, B. Jacob et al., Eigen v3, 2010, see http://eigen.tuxfamily. org/.
${ }^{76}$ PCMSolver, an Application Programming Interface for the Polarizable Continuum Model electrostatic problem, written by R. Di Remigio, L. Frediani and K. Mozgawa, see http://pcmsolver.readthedocs.org/.

${ }^{77}$ R. D. Remigio, R. Bast, L. Frediani, and T. Saue, J. Phys. Chem. A 119, 5061 (2015).

${ }^{78}$ M. Bugeanu, R. Di Remigio, K. Mozgawa, S. S. Reine, H. Harbrecht, and L. Frediani, Phys. Chem. Chem. Phys. 17, 31566 (2015).

${ }^{79}$ K. Aidas, C. Angeli, K. L. Bak, V. Bakken, R. Bast, L. Boman, O. Christiansen, R. Cimiraglia, S. Coriani, P. Dahle, E. K. Dalskov, U. Ekström, T. Enevoldsen, J. J. Eriksen, P. Ettenhuber, B. Fernández, L. Ferrighi, H. Fliegl, L. Frediani, K. Hald, A. Halkier, C. Hättig, H. Heiberg, T. Helgaker, A. C. Hennum, H. Hettema, E. Hjertenæs, S. Høst, I.-M. Høyvik, M. F. Iozzi, B. Jansík, H. J. Aa. Jensen, D. Jonsson, P. Jørgensen, J. Kauczor, S. Kirpekar, T. Kjærgaard, W. Klopper, S. Knecht, R. Kobayashi, H. Koch, J. Kongsted, A. Krapp, K. Kristensen, A. Ligabue, O. B. Lutnæs, J. I. Melo, K. V. Mikkelsen, R. H. Myhre, C. Neiss, C. B. Nielsen, P. Norman, J. M. H. Olsen, A. Osted, M. J. Packer, F. Pawlowski, T. B. Pedersen, P. F. Provasi, S. Reine, Z. Rinkevicius, T. A. Ruden, K. Ruud, V. V. Rybkin, P. Sałek, C. C. M. Samson, A. S. de Merás, T. Saue, S. P. A. Sauer, B. Schimmelpfennig, K. Sneskov, A. H. Steindal, K. O. Sylvester-Hvid, P. R. Taylor, A. M. Teale, E. I. Tellgren, D. P. Tew, A. J. Thorvaldsen, L. Thøgersen, O. Vahtras, M. A. Watson, D. J. D. Wilson, M. Ziolkowski, and H. Ågren, Wiley Interdiscip. Rev.: Comput. Mol. Sci. 4, 269 (2014).

${ }^{80}$ P. J. Stephens, F. J. Devlin, C. F. Chabalowski, and M. J. Frisch, J. Phys. Chem. 98, 11623 (1994).

${ }^{81}$ A. D. Becke, J. Chem. Phys. 98, 5648 (1993).

${ }^{82}$ T. H. Dunning, J. Chem. Phys. 90, 1007 (1989).

${ }^{83}$ B. Mennucci, E. Cancès, and J. Tomasi, J. Phys. Chem. B 101, 10506 (1997).

${ }^{84}$ M. J. Frisch, G. W. Trucks, H. B. Schlegel, G. E. Scuseria, M. A. Robb, J. R. Cheeseman, J. A. Montgomery, Jr., T. Vreven, K. N. Kudin, J. C. Burant, J. M. Millam, S. S. Iyengar, J. Tomasi, V. Barone, B. Mennucci, M. Cossi, G. Scalmani, N. Rega, G. A. Petersson, H. Nakatsuji, M. Hada, M. Ehara, K. Toyota, R. Fukuda, J. Hasegawa, M. Ishida, T. Nakajima, Y. Honda, O. Kitao, H. Nakai, M. Klene, X. Li, J. E. Knox, H. P. Hratchian, J. B. Cross, V. Bakken, C. Adamo, J. Jaramillo, R. Gomperts, R. E. Stratmann, O. Yazyev, A. J. Austin, R. Cammi, C. Pomelli, J. W. Ochterski, P. Y. Ayala, K. Morokuma, G. A. Voth, P. Salvador, J. J. Dannenberg, V. G. Zakrzewski, S. Dapprich, A. D. Daniels, M. C. Strain, O. Farkas, D. K. Malick, A. D. Rabuck, K. Raghavachari, J. B. Foresman, J. V. Ortiz, Q. Cui, A. G. Baboul, S. Clifford, J. Cioslowski, B. B. Stefanov, G. Liu, A. Liashenko, P. Piskorz, I. Komaromi, R. L. Martin, D. J. Fox, T. Keith, M. A. Al-Laham, C. Y. Peng, A. Nanayakkara, M. Challacombe, P. M. W. Gill, B. Johnson, W. Chen, M. W. Wong, C. Gonzalez, and J. A. Pople, Gaussian 03, Revision B.05, Gaussian, Inc., Wallingford, CT, 2004.

${ }^{85}$ A. H. Steindal, K. Ruud, L. Frediani, K. Aidas, and J. Kongsted, J. Phys. Chem. B 115, 3027 (2011).

${ }^{86}$ F. Goujon, P. Malfreyt, A. Boutin, and A. H. Fuchs, J. Chem. Phys. 116, 8106 (2002).

${ }^{87}$ D. Michael and I. Benjamin, J. Phys. Chem. B 102, 5145 (1998). 